Top Magn Reson Imaging. 2016 October ; 25(5): 223-235. doi:10.1097/RMR.0000000000000100.

\title{
Metabolic Imaging in Humans
}

\author{
Taylor L. Fuss, B.A. ${ }^{*}$ and Leo L. Cheng, Ph.D. ${ }^{*} \dagger$ \\ *Departments of Radiology and Pathology, Massachusetts General Hospital, Harvard Medical \\ School, Boston, Massachusetts, 02114 USA
}

\begin{abstract}
Metabolic imaging enhances understanding of disease metabolisms and holds great potential as a measurement tool for evaluating disease prognosis and treatment effectiveness. Advancement of techniques, such as magnetic resonance spectroscopy (MRS), positron emission tomography (PET), and mass spectrometry (MS), allow for improved accuracy for quantification of metabolites and present unique possibilities for use in clinic. This article reviews and discusses literature reports of metabolic imaging in humans published since 2010 according to disease type, including cancer, degenerative disorders, psychiatric disorders, and others, as well as the current application of the various related techniques.
\end{abstract}

\section{Keywords}

metabolic imaging; magnetic resonance spectroscopy imaging (MRSI); humans

\begin{abstract}
Metabolites are the small molecule intermediates and products associated with metabolic pathways. Physiological and pathological processes in an organism, such as diseases, activate or deactivate these pathways and result in changes of metabolite concentrations. At present, metabolic imaging uses techniques including magnetic resonance spectroscopy (MRS), positron emission tomography (PET), and mass spectrometry (MS) imaging to visualize and quantify metabolites, and provide a unique perspective to assist disease diagnosis, evaluate patient prognostication, and monitor treatment effectiveness.
\end{abstract}

MRS is a radiation free technique that can be used in tandem with MR imaging (MRI) to noninvasively characterize tissue with signals from hydrogen protons $\left({ }^{1} \mathrm{H}\right)$ and other nuclei, such as carbon-13. PET detects pairs of gamma rays emitted by an injected positronemitting radiotracer that is retained at disease foci through characteristic pathological processes. Depending on the radiotracer injected, different metabolic pathways can be observed and probed. MS imaging (MSI), a newly developed imaging modality, follows the traditional MS concept of ionizing chemicals with electrons and sorting them by mass-tocharge $(\mathrm{m} / \mathrm{z})$ ratio to identify the presence of molecules, but accomplishes these processes on the surfaces of tissue, rather than in the traditional gas or liquid phases. While still in its

\footnotetext{
${ }^{\dagger}$ Corresponding Author: Leo L. Cheng, PhD, $14913^{\text {th }}$ Street, CNY-6, Charlestown, MA 02129, Ph.617-724-6593, Fax. 617-726-5684, cheng@nmr.mgh.harvard.edu.

Competing Interests: The authors acknowledge no competing financial interests.

Author Contributions: Fuss: research, writing; Cheng: research, writing, funding.
} 
infancy and can suit only for ex vivo analyses, MSI presents the potential of quantifying tissue molecules and metabolites at a dimension close to single-cell metabolite measurements.

Compared with PET, which allows only for observation of metabolites related to the chosen radiotracer, and MSI, which permits only ex vivo measurements, MRS allows for both ex vivo and in vivo measurements of a complete spectrum of measurable metabolites and, therefore, occupies a prominent place in metabolic imaging. Furthermore, for in vivo evaluations, since the brain is the most suitable organ for MR evaluation, due to its relative homogeneity in magnetic susceptibility and its relative lack of respiratory and cardiac movements compared with other organs, most in vivo metabolic imaging researches involve applications of MRS with brain as the organ of interest. As a result, the majority of in vivo metabolic imaging research has been focused on diseases such as neurodegenerative diseases, psychiatric disorders, and brain cancer, the major topics reviewed in this article.

We review literature data published since 2010 and discuss research studies of metabolic imaging applications for disease diagnosis and prognosis in humans with techniques including MRS, PET, and MSI. Readers interested in earlier related topics are referred to review articles seen in literature ${ }^{1,2}$.

\section{Degenerative Disorders}

Aging is an inevitable biological process that brings with it an increase in incidence of degenerative disorders. Aging related metabolite levels vary differently for different brain regions ${ }^{3-5}$. The pons is an important part of the brainstem containing various motor and sensory nuclei and bundles. Many neurodegenerative diseases, such as Parkinson's disease, are associated with the pons. Proton MRS data of in vivo metabolite levels for normal pons has been developed to investigate pontine lesions ${ }^{6}$. Seventy-eight healthy subjects with ages from 5 days to 78 years underwent the MR measurements of turbo spectroscopic imaging (TSI), in which a fast spin-echo is applied to obtain MRSI data rapidly without the loss of spatial resolution. The metabolite ratios measured showed variations with age. $\mathrm{N}$-acetylaspartate (NAA) to creatine $(\mathrm{Cr})$ ratio was low at birth but rose rapidly between $0-5$ years, and reached a peak between 10-20 years before the start of a gradual decrease. No significant NAA/Cr difference was found within the groups of 0-5 years or those older than 50 years, but the differences within the 6-20 and 21-50 age groups were significant. The choline (Cho)/Cr ratio decreased rapidly during the first five years, and continuing to decline until 20 years, when it remained stable, leading to significant differences between 0-5 and other age groups. The Cho/NAA ratio also decreased rapidly during the first five years, and continued to decline until 20 years when a reversal was observed. These TSI measured metabolite changes caution the importance of considering age factors when using metabolite levels to characterize disease. Another example of the importance of considering patient age is seen with the investigation of possible metabolic changes in the central nervous system of patients with Fabry $(n=7)$ and Gaucher $(n=8)$ diseases using MRS at 3T. Both diseases are rare progressive inherited disorders of glycosphingolipid metabolism affecting multiple organ systems ${ }^{7}$. While no significant differences were identified between the two patient groups and age-matched healthy volunteers, there were significant age-related differences

Top Magn Reson Imaging. Author manuscript; available in PMC 2017 October 01. 
observed. A significant correlation of myo-Inositol $(m-\operatorname{Ino})(p<0.01)$ was found for all three groups. NAA/Cho significantly decreased with age $(p<0.01)$ by 6,5 and $8 \%$ per decade in Gaucher patients, Fabry patients, and healthy volunteers respectively. Therefore, it is extremely important to consider the use of age-matched controls when evaluating diseaserelated metabolic changes.

Metabolite changes across a large volume of the brain in patients with Parkinson's disease (PD), a neurodegenerative disorder, were studied in connection with neurological testing 8 . MRS data showed that in grey matter within the right temporal lobe, $\mathrm{Cr}$ was significantly elevated in PD patients compared with healthy volunteers $(p=0.027)$, likely due to greater neuronal energy expenditure as compensation early in the course of PD. There were also significant decreases bilaterally in the temporal lobes for NAA/Cr (right: $p=0.019$; left: $p=$ 0.001 ) and Cho/Cr (right: $p=0.001$; left: $p=0.007$ ), as well as in the right occipital grey matter for NAA $(p=0.032)$ and NAA/Cr $(p=0.016)$. Overall in the total cerebrum grey matter, NAA/Cr was significantly reduced in PD patients $(p=0.029)$. The feasibility of 3D MRSI of the substantia nigra (SN) region for differentiation between idiopathic PD (iPD) and atypical parkinsonian syndrome (aPS) has also been investigated ${ }^{9}$. Significant differences were observed from rostral to caudal NAA/Cr ratios between iPD patients and healthy volunteers and between iPD and aPS patients $(p<0.001)$. For healthy volunteers and aPS patients, rostral NAA/Cr was greater than caudal, and this trend was reversed in iPD patients, suggesting that MRSI could enhance diagnostic capabilities for patients with unclassifiable parkinsonian type symptoms, as well as increase understanding of metabolic changes associated with disease progression.

MRS has also showed diagnostic capabilities for other neurological disorders. Through quantification of metabolite levels in the occipital lobe and posterior cingulate gyrus (PCG), MRS was used to differentiate between dementia with Lewy bodies (DLB) and Alzheimer's disease $(\mathrm{AD})^{10}$. DLB is a common form of dementia with pathological features and symptoms similar to AD and characterized by a severe sensitivity to antipsychotic medication but a clinically favorable response to cholinesterase inhibitors. In the PCG, both $\mathrm{AD}$ and DLB patients showed significantly lower concentrations of NAA, glutamate (Glu), and combined glutamate and glutamine $(\mathrm{Glx})(p<0.05)$ when compared with healthy volunteers. In the occipital lobe, however, DLB patients showed significantly decreased NAA $(p<0.001), \mathrm{Cr}(p<0.01)$, Glu $(p<0.001)$, and Glx $(p<0.001)$ compared with healthy volunteers, whereas AD patients only showed decreases in Glu $(p<0.01)$ and Glx $(p<$ 0.05). Therefore, NAA, Cr, and Glu concentrations in the occipital lobe were significantly reduced in DLB patients compared with AD patients $(p<0.05)$, and Glu concentrations, as well as NAA+Glu+Cr concentrations, had good in vivo diagnostic efficiency, with both showing $84.2 \%$ specificity and $66.7 \%$ sensitivity.

Likewise, metabolites measured with MRS have been found to correlate with pain-rating index and cognitive disorder in patients with idiopathic trigeminal neuralgia (ITN), a disorder characterized by severe unilateral pain with recurrent episodes, spontaneous or triggered by stimulation to the affected region ${ }^{11}$. Across the thalamic subregions, NAA/Cr ratios in the affected side (contralateral to the side with pain) of the posterior lateral thalamus and the posterior medial thalamus of ITN patients were significantly decreased 
compared with age-matched healthy volunteers $(p<0.05)$, with the NAA/Cr ratio in the posterior medical thalamus negatively associating with the visual analogue score $(p<0.01)$ and the disease duration $(p<0.05)$. Significant decreases in the NAA/Cr and Cho/Cr ratios in the intralaminar portion of the thalamus were also seen in ITN patients when compared with age-matched volunteers $(p<0.05)$; and the $\mathrm{Cho} / \mathrm{Cr}$ ratio positively correlating with the Montreal Cognitive Assessment score of patients with ITN $(p<0.01)$.

For progressively degenerative disorders, MRS presents an additional and powerful clinical tool of monitor disease progression with metabolite changes. Changes in NAA, Cho, and $\mathrm{Cr}$ concentrations in the entire intracranial corticospinal tract (CST) in patients with amyotrophic lateral sclerosis (ALS) showed that MRS measurable metabolites correlate with disease severity and upper motor neuron function ${ }^{12}$. Metabolite levels of NAA, Cho, and Cho/NAA in both the right and left intracranial CST were significantly different between ALS patients and healthy volunteers. NAA was reduced $8.9 \%$ on the left side and $6.2 \%$ on the right side, while Cho was elevated $8.1 \%$ on the left side and $7.5 \%$ on the right side, and Cho/NAA was elevated $20.0 \%$ on the left side and $15.7 \%$ on the right side in ALS patients. Cho/NAA was significantly correlated with various measured of upper motor neuron function: in the left precentral gyrus for maximum forced vital capacity $(p=0.010)$ and right finger tap rate $(p=0.001)$, in the left centrum semiovale for maximum forced vital capacity $(p=0.023)$ and right finger tap rate $(p=0.002)$, and in the left posterior limb of internal capsule for maximum forced vital capacity $(p=0.045)$ and right foot tap rate $(p=0.0046)$. These results show a clear bilateral neurological asymmetry in metabolites along the intracranial CST in ALS patients quantifiable with MRS.

The increase of MR field strength presents increased spectral resolution, that translates into the improved capabilities of detecting and distinguishing metabolites in vivo. An exploratory in vivo 7T MRS study characterized metabolite changes with respect to disease stage and pathophysiological changes in Huntington's disease (HD) in three tested groups: premanifest gene carriers, manifest HD patients, and healthy volunteers (Figure 1$)^{13}$. In the caudate nucleus and the putamen, manifest HD patients showed significantly reduced NAA ( $p=0.009$ and 0.024$)$ and $\mathrm{Cr}(p=0.001$ and 0.027$)$, compared with healthy volunteers. However, no significant differences were found in the other three measured regions: hypothalamus, thalamus, or prefrontal region. Although there was no significant difference found between premanifest gene carriers and healthy volunteers, absolute values of NAA, $\mathrm{Cr}$, and Glu were lower in the caudate nucleus and putamen in premanifest gene carriers. The lower concentrations of NAA and Cr could indicate deficits in neuron integrity and energy metabolism in both manifest and premanifest HD patients; and MRS, thus, can be used as a tool to monitor disease progression and treatment effectiveness.

MRSI allows the collection of spectral data from many voxels. Multiple sclerosis (MS) disease was studied with such a localized MRSI with a coronal slice passing through the CST to investigate metabolic changes along the entire motor pathway from the internal capsule to the corona radiata ${ }^{14}$. Relapsing-remitting MS patients had significantly higher Cho concentrations in the CST than healthy volunteers $(p=0.039)$ and, although not statistically significant, trended towards higher Cho concentrations than primary-progressive MS patients $(p=0.081)$. Across all MS patients, lower CST Cho concentrations were 
correlated with worsened walking ability measured by timed walk test scores, with a mean increase in Cho concentration of $1.538 \mathrm{mmol} / \mathrm{l}$ [Regression coefficient (95\% CI) 1.538 (0.064, 3.011), $p=0.041]$ per unit of higher inverse timed walk test (in 1 per second). For primary-progressive MS patients, lower concentrations of CST Cho and Cr were associated with higher expanded disability status scale; with each expanded disability status scale point increase, there was a decrease of Cho concentration by $0.348 \mathrm{mmol} / 1$ [regression coefficient (95\% CI) $-0.348(-0.602,-0.094), p=0.007]$ and a decrease in Cr concentration by 0.990 $\mathrm{mmol} / 1$ [regression coefficient $(95 \% \mathrm{CI})-0.990(-1.841,-0.138), p=0.023$ ]. Relapsingremitting MS patients also had significantly higher CST inositol (Ino) concentrations than primary-progressive MS patients $(p=0.037)$ and a trend towards elevated Ino concentrations compared with healthy volunteers $(p=0.087)$. In relapsing-remitting MS patients, these higher Ino concentrations were associated with worsened walking ability, with an increase in Ino concentration of $17.08 \mathrm{mmol} / \mathrm{l}$ per unit of lower inverse timed walking test score (in $1 / \mathrm{s}$ ) [regression coefficient $(95 \%$ CI $)-17.08(-31.95,-2.22), p=0.024$ ]. A combination of MRS with diffusion tensor imaging (DTI) is used for evaluation of tumefactive demyelination (TD) lesions in the brain ${ }^{15}$. TD lesions, presented in MRI as a large single lesion with marked edema, can be difficult to differentiate from other brain pathologies such as neoplasm. MRS data alone can also be uncertain. For instance, among eight patients with typical morphology on MRI, seven lesions showed higher Cho, NAA, and $\mathrm{Cr}$ at the periphery of the lesion compared to the center, while for other two cases with atypical morphology observed on MRI, higher peripheral NAA, but varied Cho between the center and periphery were observed on MRS. Nevertheless, DTI revealed three distinct zones with varying diffusion characteristics in 15 patients. There was a well-defined outer zone of restricted diffusion, with a gradual increase in diffusion moving inwards towards the center, where facilitated diffusion was observed. The combination of MRI, MRS and DTI revealed distinct features at different depths of lesions.

\section{Psychiatric Disorders}

Many MR-measurable metabolites are active neurotransmitters, known to play important roles in psychiatric disorders. Post-traumatic stress disorder (PTSD) patients were found to have lower concentrations of $\gamma$-aminobutyric acid (GABA) in the lateral temporal and parieto-occipital cortices, lower NAA in the prefrontal cortex, and higher Glu in the lateral temporal cortex ${ }^{16}$. Since alcohol use disorder is frequently linked with PTSD, MRS was used to investigate the effects of heavy drinking on brain metabolite concentrations in PTSD patients with current alcohol-use-disorder $(\mathrm{n}=10)$, and compared with PTSD-only patients $(\mathrm{n}=28)$, as well as with non-PTSD volunteers $(\mathrm{n}=20)^{17}$. PTSD patients with alcohol-usedisorder had normal GABA and Glu levels on both the lateral temporal and parieto-occipital cortices, while PTSD-only patients showed higher Glu in the lateral temporal cortex ( $p=$ $0.009)$ and lower GABA in the parieto-occipital cortex $(p=0.026)$. The normal lateral temporal GABA level in PTSD patients with alcohol-use-disorder was positively associated with processing speed (Trail Making Test-A: $r=0.87, p=0.019$ ), suggesting that alcohol use could serve as a self medication to partially neutralize neurotransmitter imbalance. In the anterior cingulate cortex, PTSD patients with alcohol-use-disorder have lower Glu ( $p \leq$ $0.001)$ and higher GABA levels than both PTSD only patients and non-PTSD volunteers ( $p$

Top Magn Reson Imaging. Author manuscript; available in PMC 2017 October 01. 
$\leq 0.027$ ), while PTSD only patients had normal Glu and GABA levels. The lower concentration of Glu in PTSD patients with alcohol-use-disorder was strongly related to divided attention (Trail Making Test-B: $r=0.73, p=0.025$ ) while the higher GABA concentration was strongly related to auditory-verbal learning/memory (Hopkins Verbal Learning Test-Revised-Total Recall: $r=0.69, p=0.040$; Hopkins Verbal Learning TestRevised-Delay Recall: $r=0.89, p=0.002$ ). These results emphasize the potential impact of metabolite imaging on understanding, monitoring, and managing psychiatric disorders in clinic.

To monitor and manage psychiatric disorders, it is important to evaluate risk factors of patients before serious symptoms emerge that can pose a threat to themselves and others. Adolescents and young adults of schizophrenia patients are considered of having high familial risks of developing schizophrenia. The 23 high-risk subjects and 24 age and sex matched healthy volunteers were studied with $\mathrm{MRS}^{18}$. In comparison to healthy volunteers, NAA levels in the high-risk subjects were reduced in the thalamus, caudate, and anterior cingulate gyrus, with thalamic and anterior cingulate NAA levels inversely correlating with schizotypy. The anterior cingulate NAA concentrations also inversely correlated with attenuated psychosis and perseverative errors on the Wisconsin Card Sorting test. Glx concentrations in high-risk subjects were elevated in the thalamus and caudate and positively correlated with schizotypy and attenuated psychosis, with caudate Glx concentrations also correlated with perseverative errors in the Wisconsin Card Sorting test.

Glycerophosphocholine (GPC) and phosphocholine (PC) levels were increased in the anterior cingulate cortex compared with healthy volunteers, which positively correlated with schizotypy and attenuated psychosis. These results suggest that reduced NAA and increased Glx and Cho in the thalamus might represent vulnerability for developing schizophrenia.

Various studies have attempted to characterize bipolar disorder through metabolite imaging. One such study used MRSI to measure NAA, Cho, and $m$-Ino in the hippocampal complexes and brainstem of 19 patients with euthymic biopolar I disorder medicated with mood stabilizers and compared them with eight healthy volunteers ${ }^{19}$. Statistical analysis with Mann-Whitney U tests showed significantly increased Cho concentration in the thalamus (left: $p=0.003$; right: $p=0.02$ ), as well as significantly increase NAA concentrations in the left hippocampus $(p=0.01)$ in bipolar patients. Further analyses using one-way analysis of variance (ANOVA) revealed similar results, with significantly increased Cho concentration in the thalamus (left: $p=0.006$; right: $p=0.007$ ), as well as significantly increase NAA concentrations in the left hippocampus $(p=0.014)$ in bipolar patients compared with healthy controls. Proton MRSI study has also been used to investigate metabolic changes in both unmedicated bipolar depression $(\mathrm{n}=12)$ and mania $(\mathrm{n}=12)$, as well as 20 healthy volunteers ${ }^{20}$. Compared with healthy volunteers, bipolar disorder patients had significantly higher $\mathrm{Lac} / \mathrm{Cr}$ ratio $(p=0.03)$. Post-hoc pair-wise analysis showed that bipolar manic patients had lower $\mathrm{Glx} / \mathrm{Cr}$ levels in the posterior cingulate cortex $(p=0.013)$ and bipolar depressive patients had higher $\mathrm{Glx} / \mathrm{Cr}$ levels in the left thalamus $(p=0.023)$ when compared with healthy volunteers. Bipolar manic patients had significantly decreased $\mathrm{Glu} / \mathrm{Cr}$ levels in the posterior cingulate cortex compared with both bipolar depressive patients $(p=0.022)$ and healthy volunteers $(p=0.0002)$. Therefore, glutamate metabolism may likely play a significant role in bipolar disorder as revealed by MRSI. MRSI has also been employed to 
evaluate metabolite changes in psychiatric disorders by comparisons of metabolite concentrations between 16 patients with methamphetamine dependence and 10 patients with methamphetamine induced psychosis using voxels in the anterior cingulate cortex, dorsolateral prefrontal cortex, and frontal white matter (Figure 2) ${ }^{21}$. Both patient groups had significantly decreased NAA levels in the right anterior cingulate corted $(p<0.005)$ and right dorsolateral prefrontal cortex $(p<0.05)$ compared to healthy volunteers. In patients with methamphetamine induced psychosis, the relative NAA concentrations were significantly correlated with the patient's age at initial substance use, with a positive correlation in the right anterior cingulate cortex $(p<0.005)$ and a negative correlation in the left frontal white matter $(p<0.01)$. The methamphetamine dependent group also had decreased Cho metabolites in the right dorsolateral prefrontal cortex $(p<0.05)$ compared with healthy volunteers.

2D multivoxel MRS was also used to evaluate metabolite concentrations in the prefrontal lobe, anterior cingulate cortex, and the hippocampus of 26 patients with first-episode, treatment-naive, non-late-life major depressive disorder (MDD) together with 13 healthy volunteers $^{22}$. Patients with major depressive disorder had significantly reduced NAA/Cr ratio in the left prefrontal lobe $(p=0.033)$ and significantly reduced NAA/Cr and $\mathrm{Cho} / \mathrm{Cr}$ in the right prefrontal lobe $(p<0.05)$ compared with healthy volunteers. Patients with major depressive disorder also had significantly decreased thyroid-stimulating hormone levels compared to healthy volunteers, which positively correlated with the NAA/Cr ratio ( $p=$ 0.045 ), suggesting that biochemical abnormalities and thyroid dysfunction may begin early in major depressive disorder.

Evaluation of the effects of treatment on psychiatric disorders can also be monitored with metabolic imaging. Effects of cognitive-behavioral therapy, a highly effective therapy for pediatric obsessive-compulsive disorder (OCD), were evaluated with MRSI at 1.5T for metabolite levels in the brain ${ }^{23}$. Five unmedicated OCD patients and nine healthy volunteers underwent MRSI of the bilateral putamen, thalamus, and pregenual anterior cingulate cortex. OCD patients were also rescanned after 12 weeks of cognitive-behavioral therapy. At baseline, NAA levels in the pregenual anterior cingulate cortex of OCD patients were $55.5 \%$ higher $(p<0.05)$, while the $\mathrm{Cr}$ in the right putamen was $31.8 \%$ lower than healthy volunteers $(15.0 \% ; p<0.01)$, all after Bonferroni correction. After cognitive-behavioral therapy, the NAA $(p<0.05)$ and $\mathrm{Cr}(23.9 \% ; p<0.05)$ in the left pregenual anterior cingulate cortex decreased in all five patients. An increase of $10.6 \%$ was also observed in the right thalamus $(p<0.01)$ in all five patients.

Additional researches have applied various metabolic imaging techniques to investigate brain metabolic changes in patients with amnestic mild cognitive impairment (aMCI). Multivoxel proton MRS measurements using a PRESS pulse sequence at $1.5 \mathrm{~T}$ for $14 \mathrm{aMCI}$ patients and 15 healthy volunteers were reported ${ }^{24}$. aMCI patients showed significant decreases in NAA $(p<0.01)$, NAA/Cr $(p<0.01)$, and NAA $/ m$-Ino $(p<0.01)$ in the left posterior cingulate gyrus compared with healthy volunteers. A decrease in NAA $(p<0.05)$ was also found in the left inferior precuneus and posterior paratrigonal white matter, and there was a significant decrease in the NAA $/ m$-Ino $(p<0.05)$ ratio in the left posterior paratrigonal white matter. Another aMCI study compared the metabolic imaging techniques 
of fluorodeoxyglucose-PET (FDG-PET) with advanced MR techniques, including MRS, perfusion weighted imaging (PWI) and diffusion tensor imaging (DTI), within the posterior cingulate region of the brain in 55 aMCI patients and 20 age-matched controls. The z-scores, values of standard deviations, was used to reflect the degree of abnormalities of patients compared to the healthy volunteers, with higher z-scores representing more pronounced alterations ${ }^{25}$. DTI had significantly higher z-scores $(2.1 ; p<0.0001)$ compared with FDGPET (0.9) and PWI (1.0) and the lowest MRS z-scores (NAA/Cr: 0.3; Cho/Cr: 0.1; m-Ino: $0.2)$. FDG-PET and PWI measurements showed significant positive correlations $(r=0.44 ; p$ $=0.001)$. Compared with healthy volunteers, aMCI patients showed significant glucose hypometabolism $(p<0.05)$. FDG-PET and PWI techniques showed similar accuracy in distinguishing between aMCI patients and healthy volunteers, while DTI had increased accuracy. Based on FDG-PET data, aMCI patients were divided into two groups: those expressing lower glucose uptake and those with normal glucose uptake. Both groups did not show any significant differences in MRS-measured metabolite levels, but did show significantly lower DTI values. Those with lower glucose uptake also had lower values of PWI cerebral blood volume.

\section{Other Neurological Disorders}

Metabolic imaging has also been utilized to understand the metabolic changes associated with other disorders. Multi-voxel MRS was used to investigate possible differences in metabolite ratios between mild $(n=17)$ and severe $(n=14)$ obstructive sleep apnea patients ${ }^{26}$. Compared with patients with mild obstructive sleep apnea, patients categorized as severe had significantly elevated NAA/Cr $(p=0.004)$ and $\mathrm{Cho} / \mathrm{Cr}(p=0.003)$ ratios in the hippocampus. In the putamen, $\mathrm{Cho} / \mathrm{Cr}(p=0.004)$ was again elevated while NAA/Cho ( $p=$ 0.032) was decreased in patients with severe obstructive sleep apnea. Metabolic differences in the hippocampi of patients with juvenile myoclonic epilepsy $(n=17)$ versus age and sex matched healthy volunteers $(n=19)$ were also studied with MRS at $1.5 T^{27}$. Statistical analysis with ANOVA $(p<0.05)$ revealed significant differences between groups for $\mathrm{Cho} / \mathrm{Cr}$ in the head of the hippocampus, NAA/Cr in the head, body, and tail of the hippocampus, and $\mathrm{NAA} / \mathrm{Cho}+\mathrm{Cr}$ in the body and tail of the left and right hippocampus. However, none of the significant metabolite differences correlated with duration of epilepsy. These results suggest the metabolite changes are a part of disease pathology and further investigation could help improve understanding of disease and enhance treatment.

Additional research has used imaging to evaluate the effects of environment on metabolite levels. MRS was used to examine the association between mean childhood blood lead levels and in vivo brain metabolite concentrations as adults ${ }^{28}$. Participants came from the Cincinnati Lead Study $(n=159)$ and had a mean childhood blood lead values of $13.3 \pm 6.1$ micrograms per deciliter ( $\mu \mathrm{g} / \mathrm{dL}$ ), ranging from 4.7 to $37.2 \mu \mathrm{g} / \mathrm{dL}$. Using a model, an increase in mean childhood blood lead level concentration was associated with a decrease in NAA and $\mathrm{Cr}$ in the basal ganglia, a decrease in NAA and Cho in the cerebellum, a decrease in Glx in the vermis and parietal white matter, and a decrease in Cho in the parietal and frontal white matter. MRS also detected rf changes in metabolite levels in the cerebellum after heat stroke ${ }^{29}$. In patients $(\mathrm{n}=8)$ after heat stroke, NAA/Cr $(p=0.004)$ and $\mathrm{Cho} / \mathrm{Cr}(p=$ 0.032 ) ratios were significantly decreased compared with healthy volunteers. The NAA/Cr

Top Magn Reson Imaging. Author manuscript; available in PMC 2017 October 01. 
ratio was also significantly correlated with the patient's Glasgow Coma Scale score $(r=$ $0.748 ; p=0.033$ ), suggesting that the severity of heat stroke can be effectively evaluated in clinic by NAA/Cr ratios measured in vivo with MRS.

\section{Brain Cancer}

Cancer is a major public health threat worldwide with 14.4 million new cases and 8.2 million deaths in $2012^{30}$. With the increased emphasis on the clinical value of early detection and treatment, imaging diagnostic efforts have focused extensively on the biological characterization of cancer, assisted by genomics, proteomics, and metabolomics. Evaluations of disease-related alterations in metabolite levels can provide valuable insight into individual cancer metabolism and present the potential to inform diagnosis and treatment management in the clinic, all align with the goals of individualized precision medicine.

Most brain tumors are detected using MR imaging and diagnosed with histopathological evaluation of surgically removed tissue to confirm cancer type and grade. Major cancer metabolite imaging developments have occurred with in vivo MRS studies of brain cancer. While brain cancer is not the most prevalent cancer in adults, with 256,000 new cases and 189,000 deaths in 2012, the highest incidence and mortality rates are found in more developed regions such as Australia/ New Zealand, Europe, and North America, likely reflecting the importance of available diagnostic imaging facilities for accurate diagnosis and treatment ${ }^{30}$.

Metabolite imaging of brain tumors aims at developing non-invasive diagnostic and treatment evaluation protocols and has been used to acquire both MRS measurements of single anatomic locations and in two or three physical dimensional MRS maps. As a major type of human brain tumors, gliomas have been extensively studied by in vivo MRS for characterizations. One such study evaluated the usefulness of MRS in differentiating brain metastases $(n=14)$, from primary high-grade (HGG; $n=32)$, and low-grade (LGG; $n=14$ ) gliomas in 60 histologically confirmed patients ${ }^{31}$. Statistical analyses compared the metabolite ratios within these tumors and their peritumoral edema using the Wilcoxon tests at a significance level of $5 \%$. The metabolite ratios of $\mathrm{Cho} / \mathrm{Cr}, \mathrm{Cho} / \mathrm{NAA}$, and NAA/Cr measured from the tumoral cores showed statistically significant $(\mathrm{p}<0.05)$ differences for HGG versus metastases, LGG versus metastases, and LGG versus HGG. However, for peritumoral edema, the mean ratios of $\mathrm{Cho} / \mathrm{Cr}$, Cho/NAA, and NAA/Cr were only able to significantly differentiate between LGG and HGG. Lipid levels were also found to be significantly higher in LGG and HGG compared with metastases. Lactate levels could also be used to differentiate HGG from LGG and metastases. These data suggest that MRS could be used as a non-invasive diagnostic tool for patients who cannot undergo biopsy and for all patients suspected of harboring brain tumors as preliminary investigative procedures. Evaluations of the efficiency of the combination of three dimensional (3D) MRI textural features and MRS metabolite ratios using pattern recognition methods has been carried out to distinguish between meningiomas $(\mathrm{n}=19)$ and metastatic brain tumors $(\mathrm{n}=21)^{32}$. Brain MR images with corresponding spectral data for Cho, NAA, and $\mathrm{Cr}$, as well as the ratios Cho/NAA, Cho/Cr, and NAA/Cr for these patients were investigated against their MRI 
textural features, such as kurtosis, entropy, difference entropy, run length nonuniformity, and long run emphasis. The results presented a high classification accuracy of $95 \%$ in discriminating between meningiomas and metastases, with individual accuracies classifying meningiomas at $94.74 \%$ and metastatic brain tumors at $95.24 \%$. Analyzing only metabolites observed during spectroscopy led to a slightly lower classification accuracy of $90 \%$ for distinguishing between meningioma and metastases, with individual accuracies classifying meningioma at $89.47 \%$ and metastatic tumors at $90.48 \%$. When both features were combined, the overall classification accuracy was $100 \%$. This suggests that supplementing MRI data with metabolite information might increase the discrimination accuracy of pattern recognition systems and function as a useful tool for clinicians.

Additional research investigated the characteristics of gliomas presented with a genetic mutation of arginine 132 in the enzyme isocitrate dehydrogenase-1 (IDH1). This mutation produces an excess of the metabolite 2-hydroxyglutarate (2HG) and is seen in up to $86 \%$ of grade II and III gliomas ${ }^{33}$. Since patients with IDH1 mutations, after correcting for the age factor, have a greater 5-year survival than those without the mutation, identifying patients with this mutation could help inform diagnosis and treatment in the clinic. 2HG can be measured with in vivo MRS. 3T MRS 2HG measurements for two glioma and four primary glioblastoma (IV) patients, all with known IDH1 mutations, and four healthy volunteers were shown in Figure 3. In patients with IDH1 mutations, the $\mathrm{H}_{\mathrm{a}}-\mathrm{H}_{\beta}$ cross-peaks of $2 \mathrm{HG}$ were present at 4.02/1.91 ppm in a two-dimensional (2D) spectrum, providing clear evidence of elevated 2HG levels that, due to chemical shift overlap, is hard to identify within 1D spectra. As expected, $2 \mathrm{HG}$ cross-peaks do not appear in 2D spectra from tumor patients without IDH1 mutations and healthy volunteers. Since the acquisition time for an in vivo 2D spectrum with a voxel size of $3 \times 3 \times 3 \mathrm{~cm}^{3}$, a concentration of $2 \mathrm{mM}$, and a signal-to-noise ratio of 5 was about 13 minutes, it is conceivable that translation of such a 2D MRS into the clinic could advance diagnosis and inform treatment. $2 \mathrm{HG}$ levels can also been rapidly detected in tissue samples from surgically resected gliomas to immediately identify IDH1 mutations, using desorption electrospray ionization (DESI) MS. It has been demonstrated that during two surgical resections, by applying miniscule amounts of biopsy material onto a standard glass slide with either a swab and by smearing the tissue between two glass slides. Within minutes, mass spectrometry data revealed peaks corresponding to $2 \mathrm{HG}(\mathrm{m} / \mathrm{z} 147.0)$, which was confirmed later after surgery with immunohistochemistry (Figure 4$)^{34}$. These results show that $2 \mathrm{HG}$ can be rapidly detected using DESI MS during surgery without complex tissue preparation, allowing for rapid molecular characterization. With real-time characterization, DESI MS could help define surgical margins, leading to more balanced surgical strategy and potentially longer patient survival.

MRSI has also been used to evaluate the effectiveness of radiotherapy for brain tumors, as well as the possible radiation damage to the brain tissue. Metabolite changes in the brain before and after radiotherapy for nasopharyngeal cancer have been compared to reveal the outcome of brain radiation injury and possible repair mechanisms ${ }^{35}$. Forty-seven nasopharyngeal cancer patients underwent MRI and MRS at $1.5 \mathrm{~T}$ before radiotherapy and at each dose of 20, 40, and $60 \mathrm{~Gy}$, and with MRS follow-up at 6 and 12 months post completion of radiotherapy. Compared with metabolite levels prior to radiotherapy, the three measured metabolite concentration ratios of $\mathrm{NAA} / \mathrm{Cr}$, $\mathrm{Cho} / \mathrm{Cr}$, and $\mathrm{NAA} / \mathrm{Cho}$ were all 
reduced at the end of radiotherapy. However, these ratios steadily rebounded from the fourth through twelfth month post radiotherapy. At month 12 , the $\mathrm{Cho} / \mathrm{Cr}$ and NAA/Cho ratios were only slightly lower than their pre-radiotherapy values and, when compared with levels immediately after the completion of radiotherapy, all three ratios were significantly increased by $24.46 \%, 13.39 \%$, and $30.39 \%$, respectively.

In vivo MRS metabolic imaging can be achieved with various techniques; and the utilization of an appropriate approach is critical for a particular clinical application. Among all the available approaches, the use of single voxel spectroscopy (SVS) or multi-voxel MRS imaging (MRSI) are the two most fundamental elections that need to be determined. A comparative study of SVS and MRSI at $1.5 \mathrm{~T}$ for 36 pediatric patients with diffuse intrinsic pontine glioma between 1 and 21 years of age was conducted ${ }^{36}$. SVS is faster with less than five minutes of acquisition time and provides an assessment of all the measurable metabolites of tumor within a selected volume. MRSI, on the other hand, requires longer acquisition and processing times, but produces metabolic profiles from multiple voxels containing tumor and surrounding tissue. For the two metabolite ratios evaluated, Cho/NAA and $\mathrm{Cho} / \mathrm{Cr}$, there were strong linear correlations between SVS and MRSI data of $r=0.67$ and $r=0.76$, respectively ( $\mathrm{p}<0.0001$ for each). Overall, SVS is most suitable for assessments of tumor metabolism before and after therapy, while MRSI could be best applied to evaluate targeted therapies and monitor surrounding areas for possible tumor invasion.

The utility of combining functional MR methods of diffusion tensor imaging (DTI) and MRS has also been compared with evaluations of the peritumoral area of cerebral gliomas in 45 histology-confirmed patients ${ }^{37}$. A gradual increase in the DTI measurements of fractional anisotropy (FA), an assessment of white matter organization, and NAA/Cr ratios and a gradual decrease in the $\mathrm{Cho} / \mathrm{Cr}$ and lactate/lipids (LL)/Cr ratios were observed from the tumor center to the peritumoral zone for the four regions of interest measured (tumor center, perifocal edema, distant normal-appearing white matter, and contralateral normal-appearing white matter). The differences were significant when comparing mean FA $(p<0.001)$ and mean LL/Cr values for all regions $(\mathrm{p}<0.05)$. The $\mathrm{Cho/Cr}$ ratio was significantly different between tumor center and both distant and contralateral normal-appearing white matter $(p<$ $0.001)$, between perifocal edema and distant normal-appearing white matter $(p=0.013)$, and between perifocal edema and contralateral normal-appearing white matter $(p=0.011)$. Differences in NAA/Cr ratios between regions were not significant. The measured repeatability index $r$ was measured with a single factor ANOVA and showed high repeatability for FA values and $\mathrm{LL} / \mathrm{Cr}$ and $\mathrm{Cho} / \mathrm{Cr}$ ratios. The $\mathrm{LL} / \mathrm{Cr}$ ratio and $\mathrm{FA}$ value may present the best option for detecting the extent of glial tumors in vivo with high repeatability, and the $\mathrm{Cho} / \mathrm{Cr}$ ratio could be best utilized in presenting additional information about the perifocal edema zone, where there is a high concentration of tumor cells. Thus, varying techniques used in concert can provide complementary information that could be useful in diagnosing and monitor disease in clinic.

In addition to utilizing MR approaches alone, recently, the feasibility of simultaneous acquisition of MRS and PET for the purposes of grading tumors and examining spatial distributions of metabolic changes in glioma in vivo has been tested using MR-compatible 
PET detectors mounted on modified 3T whole-body MRI scanners ${ }^{38}$. Twenty-eight patients with histologically confirmed gliomas (16 LGG and 12 HGG) underwent simultaneous methionine (Met) MR-PET to identify the most malignant tumor part before surgical interventions. Tumor to normal brain (T/N) Met uptake ratios and the metabolite peaks, Cho, $\mathrm{Cr}$, and NAA, were measured. The median Met uptake was significantly different between LGG and HGG ( $p=0.01$ ), with values of 1.4 (range, 1-2.0) and 2.3 (range, 1.7-3), respectively. The Spearman rank correlations of metabolic markers in LGG showed a significant correlation between Met uptake and $\mathrm{Cr} / \mathrm{NAA}(p=0.015)$, as well as between Cho/NAA and $\mathrm{Cr} / \mathrm{NAA}$ ratios $(p=0.0002)$. High expression of amino acid transporters was showed in two patients with LGG through the T/N Met uptake ratio, indicative of proliferating cell populations, but didn't spatially correlate with neuronal cell loss and cell membrane proliferation, measured via Cho/NAA with MRS. Increased Cr/NAA was associated with increased Met uptake in LGG. Combined MR-PET imaging presented itself as a useful clinical tool in diagnosis and pre- surgical planning.

\section{Metabolic Imaging Beyond the Brain}

As MR methodologies improve, including increases in field strength, designs of new coils, and implementation of advanced pulse sequences, organs other than the brain have entered the focuses of MRS-based metabolic imaging. Most noticeably, the development of internal endorectal receiving coils used in combination with external phased-array coils has led to increased signal intensity and spatial resolution seen with in vivo MRS for patients with prostate cancer (PCa). Since the early 2000's, the combination of quantitative dynamic contrast-enhanced (DCE) MRI with 2D MRSI ${ }^{39}$ and creation of pulse sequences to control $J$-modulation and allow citrate to remain in phase in spectra with a reasonable echo time ${ }^{40}$ have allowed the use of MR spectroscopic imaging (MRSI) to study PCa in vivo, with particular emphasis on evaluation of disease aggressiveness and stage ${ }^{41-43}$ and treatment response $^{44-46}$. Multi-voxel MRSI in conjunction with MRI has also showed better correlations with patient pathological Gleason grade than results from biopsy, MRI, or MRSI alone ${ }^{47}$. Fifty-two patients underwent MRI and multi-voxel MRSI 6-8 weeks post biopsy and subsequent prostatectomy. A total of 86 cancer locations were identified from step-section pathological evaluations of the entire prostates after prostatectomies. Biopsy identified 74 locations with 12 false-negative and six false positive (sensitivity of $86 \%$, specificity of $67 \%$, and accuracy of $83 \%$ ). MRI detected 72 locations (sensitivity of $84 \%$, specificity of $89 \%$, and accuracy of $85 \%$ ) and multi-voxel MRSI detected 72 locations (sensitivity of $84 \%$, specificity of $78 \%$, and accuracy of $83 \%$ ). However, multi-voxel MRSI in conjunction with MRI detected 78 locations with eight false-negative and four falsepositive (sensitivity of $91 \%$, specificity of $78 \%$, and accuracy of $88 \%$ ). Cohen's test confirmed that biopsy had a lower level of agreement with histology than multi-voxel MRSI with MRI ( 0.559 , moderate agreement, versus 0.735 , good agreement). These results suggest the possible role of MRSI as a non-invasive measure of predicting PCa aggressiveness.

Recently, MRSI was used to assess PCa aggressiveness by measuring ratios of metabolites in 43 patients with biopsy-proven $\mathrm{PCa}^{48}$. With in vivo MRSI, a larger (Cho+Cr)/citrate (Cit) ratio was determined to significantly correlate to high grade $\mathrm{PCa}(p=0.02 ; r=0.27)$, as defined by containing a Gleason grade of 4 as the primary and/or 5 as a primary/secondary 
or tertiary grade. There was also a significant difference between low grade, defined as containing only Gleason grade 2 and/or 3, and high grade tumors for the median $\mathrm{Cho} / \mathrm{Cr}$ ratio ( $p<0.01 ; r=0.31$ ), with higher grades showing a larger ratio. When measured by both in vivo MRSI and ex vivo high resolution magic angle spinning (HRMAS) MRS, a strong positive correlation between higher Gleason score and increased metabolic ratio of ( $\mathrm{Cho}+\mathrm{Cr}$ + spermine $(\mathrm{Spm})) /$ Cit also was found using Spearman's rank correlations $(\rho=0.77$ and $\rho=$ 0.69 , respectively; $p<0.001)^{49}$. Due to the higher resolution achieved using ex vivo HRMAS MRS in comparison with in vivo MRSI, the Spm peak, which is located between $\mathrm{Cho}$ and $\mathrm{Cr}$, could be differentiated from the Cho and $\mathrm{Cr}$ peaks and is therefore included in the ratio calculations, to mimic the $(\mathrm{Cho}+\mathrm{Cr})$ values measured in vivo.

MRSI has also been used to evaluate the effectiveness of various PCa treatments. One such study used a combination of DCEMR and MRSI to measure changes in metabolite levels at baseline and regular time intervals thereafter $(4,12$, and 24 weeks) during hormone therapy ${ }^{50}$. At baseline measurements, healthy prostate tissue showed higher levels of Cit and intermediate levels of $\mathrm{Cho}$ and $\mathrm{Cr}$, on the other hand, $\mathrm{PCa}$ tissue showed a reduction in Cit and an increase in Cho+Cr. During hormone therapy, while there were no significant correlations between MRSI/DCEMR variations and patient age, pretreatment serum prostate specific antigen (PSA) levels, or Gleason score, there was a significant time-dependent loss of all prostate metabolites in both healthy and PCa tissue during hormone therapy $(p<0.01)$, resulting in complete metabolic atrophy after 24 weeks of hormone therapy for $30 \%$ of patients. In PCa tissue, the loss of metabolites was delayed compared with healthy tissue, with no variation observed after four weeks and higher variation from healthy tissue observed after 24 weeks than after 12 weeks. In addition to the presence of metabolic atrophy, Cit decreased the fastest and correlated significantly $(r=0.4530 ; p<0.01)$ with serum PSA levels. Patients with detectable Cit had significantly $(p<0.05)$ higher mean PSA levels (at 24 weeks, mean: $3.5 \pm 0.5 \mathrm{ng} / \mathrm{mL}$ ) than those without detectable Cit (at 24 weeks, mean: $0.85 \pm 0.9 \mathrm{ng} / \mathrm{mL}$ ). With DCEMR, the parameters of onset-time of signal enhancement and time-to-peak significantly $(p<0.05)$ increased at 12 and 24 weeks. These results suggest that MRSI and DCEMR could be used adjuvantly with PSA levels as a noninvasive method for monitoring time-dependent efficacy of hormone therapy. Similarly, MRSI has also been used to measure metabolic atrophy (defined as $\mathrm{Cho}, \mathrm{Cr}$, and $\mathrm{Cit}$ peak area to noise ratio < 5:1) in 109 patients after hormone and external beam irradiation therapies ${ }^{51}$. Patients were divided into three groups by time elapses between external beam irradiation and the time of MRSI tests after irradiations while continuing receiving hormone therapy in the duration to achieve complete clinical response. MRSI detected metabolic atrophy in 20 out of 37 patients less than one year after irradiation (54.1\%); in 32 out of 36 patients one to two years post irradiation (88.9\%); and in 34 out of 36 patients greater than two years post irradiation (94.5\%). Since metabolic atrophy is indicative of successful treatment, as cancer growth cannot occur without metabolite activities, the results support the continued use of hormone therapy after external beam irradiation, as well as the use of MRSI to measure response status. Additionally, MRSI measured metabolic atrophy has been investigated to correlate biochemical relapse represented by increases in serum PSA levels after treatments ${ }^{52}$. Out of 50 patients measured after external beam radiation therapy, 13 patients $(27 \%)$ showed complete metabolic atrophy $(\mathrm{Cho}+\mathrm{Cr} / \mathrm{Cit}$ ratio $<0.2)$ at three 
months post therapy, which was maintained throughout subsequent follow ups at six, 12, 18, and 24 months. 35 patients showed partial response to therapy, with a $\mathrm{Cho}+\mathrm{Cr} / \mathrm{Cit}$ ratio between 0.2 and 0.8 at both 3 and 6 months. Metabolic atrophy was reached at 12 months for 30 of these patients. Five patients developed a recurrence $(\mathrm{Cho}+\mathrm{Cr} / \mathrm{Cit}$ ratio $>0.8)$ at 12 months, which led to a biochemical relapse at 18 months for three patients and 24 months for two patients. MRSI characterized metabolic changes was also used to evaluate the temporary PSA increase also known as the PSA "bounce" period, which can occur in 30$40 \%$ of patients after ${ }^{125} \mathrm{I}$ brachytherapy ${ }^{53}$. A definitive decrease in the $\mathrm{Cho}+\mathrm{Cr} / \mathrm{Cit}$ ratio was observed post therapy (Figure 5). For 21 out of 24 patients, the PSA "bounce" was benign and did not lead to recurrence. For two patients with local recurrence, a continued increase in $\mathrm{Cho}+\mathrm{Cr} / \mathrm{Cit}$ ratio and PSA levels were observed over time. The one patient with serum PSA increase but no residual cancer showed decreasing in $\mathrm{Cho}+\mathrm{Cr} / \mathrm{Cit}$ ratio. Thus, MRSI measurable metabolic activity in conjunction with PSA levels monitoring may provide more complete evaluations of treatment response for patients.

Further technology developments have also been reported by recent studies. Application of a PRESS sequence with the conformal voxel MRS (CV-MRS), a technique which automatically optimizes the placement of numerous spatial saturation planes to adapt the excitation volume to the shape of the prostate, was combined with a spectral-spatial $90^{\circ}$ radiofrequency $(\mathrm{RF})$ excitation pulse. This was tested at 1.5T, for the effect of CV-MRS, on 10 healthy volunteers using the standard disposable endorectal coil inflated with approximately $75 \mathrm{~mL}$ of FC-77 FLUORINERT, a perfluocarbon compound, to ensure that the coil remained pressed against the prostate ${ }^{54}$. By decreasing the TE from $130 \mathrm{~ms}$ to 40 $\mathrm{ms}$, the full citrate multiplet was observed, as well as the peaks for $m$-Ino, scyllo-inositol, taurine, and Glx, with a 35\% improvement in the number of peaks. Cit detection improved $42 \pm 24 \%$ over the entire prostate volume, with a range of improvements from $16 \%$ to $100 \%$. With CV-MRS, metabolites with a short TE were consistently measured while the signal-tonoise and baseline improved.

Another promising area of advancement seen with MRSI relates to the recent developments in hyperpolarization technologies. Hyperpolarized $\left[1-{ }^{13} \mathrm{C}\right]$ pyruvate has been used for in vivo ${ }^{13} \mathrm{C}$ MRSI as a noninv

\section{Methodology Development}

Developments in MRS techniques and data acquisition paradigms are continually being designed and tested mostly achieved with human volunteers. As field strength of MR clinical scanner increases, optimization of in vivo MRS at 7T for future clinical use has been attended. Optimized 7T-stimulated echo-acquisition mode (STEAM) method with variablerate selective excitation pulses has been tested for investigating regional variations in GABA, Gln, and Glu compared with metabolite distribution along cingulate subregions in healthy volunteers ${ }^{56}$. All three metabolites could be measured accurately with mean signalto-noise ratios $>30$ and mean full width at half the maximum values $<10 \mathrm{Hertz}$ for all regions except pregenual anterior cingulate cortex $(14.6 \mathrm{Hertz})$. Retest reliability was quantified and assured by intraclass correlation coefficient of $>0.75$ in all cases for all spectra completing the minimum testing concentrations for two measurements on two separate testing days. Repeated-measured ANOVA showed a significant main effect for

Top Magn Reson Imaging. Author manuscript; available in PMC 2017 October 01. 
regional metabolite concentrations without correction for grey matter content $(p<0.001)$, as well as a significant interaction effect for metabolite by brain regions $(p=0.001)$. GABA was found in significantly higher concentrations in pregenual anterior cingulate cortex than other regions $(p<0.008)$. Gln concentrations were significantly higher in the pregenual anterior cingulate cortex than in the anterior mid-cingulate cortex $(p=0.003)$, rostral posterior cingulate cortex $(p=0.001)$, and caudal posterior cingulate cortex $(p=0.001)$. Glu concentrations were also significantly higher in the pregenual anterior cingulate cortex than in the anterior mid-cingulate cortex $(p=0.001)$ and rostral posterior cingulate cortex ( $p=$ 0.010). Modified point resolved spectroscopy pulse (PRESS) at 7T in vivo has also been optimized for detection of GABA, Glu, and Gln, which overlapped in short TE MRS and made precise quantifications of these metabolites difficult ${ }^{57}$. Numerical simulations led to a PRESS sub-echo times set $\left(\mathrm{TE}_{1}, \mathrm{TE}_{2}\right)=(31,61) \mathrm{ms}$ for detection of GABA and Glu, where $\mathrm{TE}_{1}$ and $\mathrm{TE}_{2}$ are the sub-echo times of the first and second slice-selective $180^{\circ} \mathrm{RF}$ pulses, respectively. Applying the resulting PRESS TE $=92 \mathrm{~ms}$, the linewidths for both GABA and Glu multiplets between 2.2 and 2.4 ppm were narrowed and separated. GABA concentration was estimated to be approximately seven times higher in grey matter than in white matter, with overall higher concentrations in the frontal compared with the occipital brain. Glu was approximately two times higher in grey matter than in white matter in both the fontal and occipital brain. Gln was significantly different between frontal grey matter and white matter, but no significant difference was observed between occipital grey and white matter. These result indicate that the PRESS TE $=92 \mathrm{~ms}$ methods is effective for measuring GABA and nearby metabolites in vivo at $7 \mathrm{~T}$.

On the other hand, metabolic differences between white matter and grey matter have also been estimated to account for partial volumes effects when the other matter is diffused into the matter of interest ${ }^{58}$. After studying with 18 volunteers, it was found that NAA, Cr, Cho, and myo-Ino concentrations in grey matter were $8.5 \pm 0.7,6.9 \pm 0.6,1.2 \pm 0.2$, and $5.3 \pm 06$ $\mathrm{mM}$, while those in white matter were $7.7 \pm 0.6,4.9 \pm 0.5,1.4 \pm 0.1$, and $4.4 \pm 0.6 \mathrm{mM}$, respectively. Thus grey and white matter absolute quantification can vary by $5-10 \%$ when mixtures are studied.

Research work at $7 \mathrm{~T}$ also investigated whether metabolite changes in the cortex during prolonged visual stimulation correlate to blood oxygenation level-dependent functional MRI (BOLD-fMRI) with 12 healthy volunteers ${ }^{59}$. During stimulation, the changes in Asp, glucose, Gly, and Lac concentrations with a range of \pm 0.2 to $0.3 \mu \mathrm{mol} / \mathrm{g}$ were highly significant, suggesting their use as biomarkers of neuronal activation in the visual cortex. The BOLD-fMRI amplitudes during stimulation were significantly correlated positively with relative concentration changes in glucose $(r=0.73 ; p=0.01)$ and Lac $(r=0.65 ; p=0.003)$ (Figure 7). There was also a significant inverse correlation between BOLD-fMRI amplitude and the resting-state concentration of $\operatorname{GABA}(r=-0.65 ; p=0.043)$.

In addition to in vivo metabolic imaging, advances have occurred in metabolomic imaging as well. While metabolic imaging measures metabolites, metabolomics evaluates all of the measurable metabolites and their changes within a metabolome, providing a more comprehensive understanding of physiological and pathological processes, and therefore valuable insight into disease progression, than can be achieved through examinations of any 
one metabolite or pathway. One study examined the metabolomic profiles of $\mathrm{PCa}$ as measured at $7 \mathrm{~T}$ from whole prostates removed during prostatectomy ${ }^{60}$. First, metabolic regions and corresponding principal component analysis (PCA) loading factors for each region were determined by measuring human prostate biopsy cores ex vivo at $14 \mathrm{~T}$ using HRMAS MRS. Introduced in $1996^{61}$, HRMAS MRS borrows from the solid-state MRS line-narrowing concept of magic angle spinning applying a mechanical rotation of the tissue sample at a $54.7^{\circ}$ angle from the magnetic field to allow for high resolution observation of metabolites from intact tissue, which is preserved for subsequent histopathology. These loading factors for each region between 0.5 and $4.5 \mathrm{ppm}$ were applied to metabolic intensities already normalized by the spectral intensity for each voxel, which served as a malignancy index. This malignancy index, linearly correlated with lesion size, has a 93 to 97\% overall accuracy for detecting PCa lesions. This technique demonstrates the importance of better understanding metabolomic profiles ex vivo to improve biochemical understanding and applications to diagnostic technology, as well as the utility of measuring and analyzing the complete metabolomic profile to understand disease physiology.

\section{Conclusions}

Metabolic imaging can significantly contribute to understanding and characterizing different diseases, as well as natural processes such as aging. As techniques improve, MRS and PET show great potential for enhancing diagnosis in clinic, as well as monitoring treating and prognosis, while MS has already been successfully used to complement pathology for diagnosis and margin evaluation during surgery, and could be used to help inform surgical treatment.

\section{Acknowledgments}

We thank the Massachusetts General Hospital Athinoula A. Martinos Center for Biomedical Imaging for research support. Funding: This work was funded by PHS NIH grants CA115746, CA115746S2, and CA162959.

\section{Abbreviations}

\section{HG 2-hydroxyglutarate}

3D three-dimensional

AD Alzheimer's disease

ALS amyotrophic lateral sclerosis

aMCI amnestic mild cognitive impairment

ANOVA analysis of variance

aPS atypical parkinsonian syndrome (aPS)

BOLD-fMRIblood oxygenation level-dependent functional MRI

Cho choline

Cit citrate 


$\begin{array}{ll}\text { Cr } & \text { creatine } \\ \text { CST } & \text { corticospinal tract } \\ \text { CV-MRS } & \text { conformal voxel MRS } \\ \text { DCE } & \text { dynamic contrast-enhanced } \\ \text { DESI } & \text { desorption electrospray ionization } \\ \text { DLB } & \text { dementia with Lewy bodies } \\ \text { DTI } & \text { diffusion tensor imaging }\end{array}$

FA fractional anisotropy

FDG-PET fluorodeoxyglucose-PET

GABA $\gamma$-aminobutyric acid

Glu glutamate

Glx combined glutamate and glutamine

GPC glycerophosphocholine

Gy gray

HD Huntington's disease

HGG high-grade gliomas

HRMAS high resolution magic angle spinning

IDH1 isocitrate dehydrogenase-1

Ino inositol

ITN idiopathic trigeminal neuralgia

iPD idiopathic Parkinson's disease

LGG low-grade gliomas

LL lactate/lipids

Met methionine

m-Ino myo-Inositol

mM millimolar

MR magnetic resonance

MRI magnetic resonance imaging

MRS magnetic resonance spectroscopy

Top Magn Reson Imaging. Author manuscript; available in PMC 2017 October 01. 


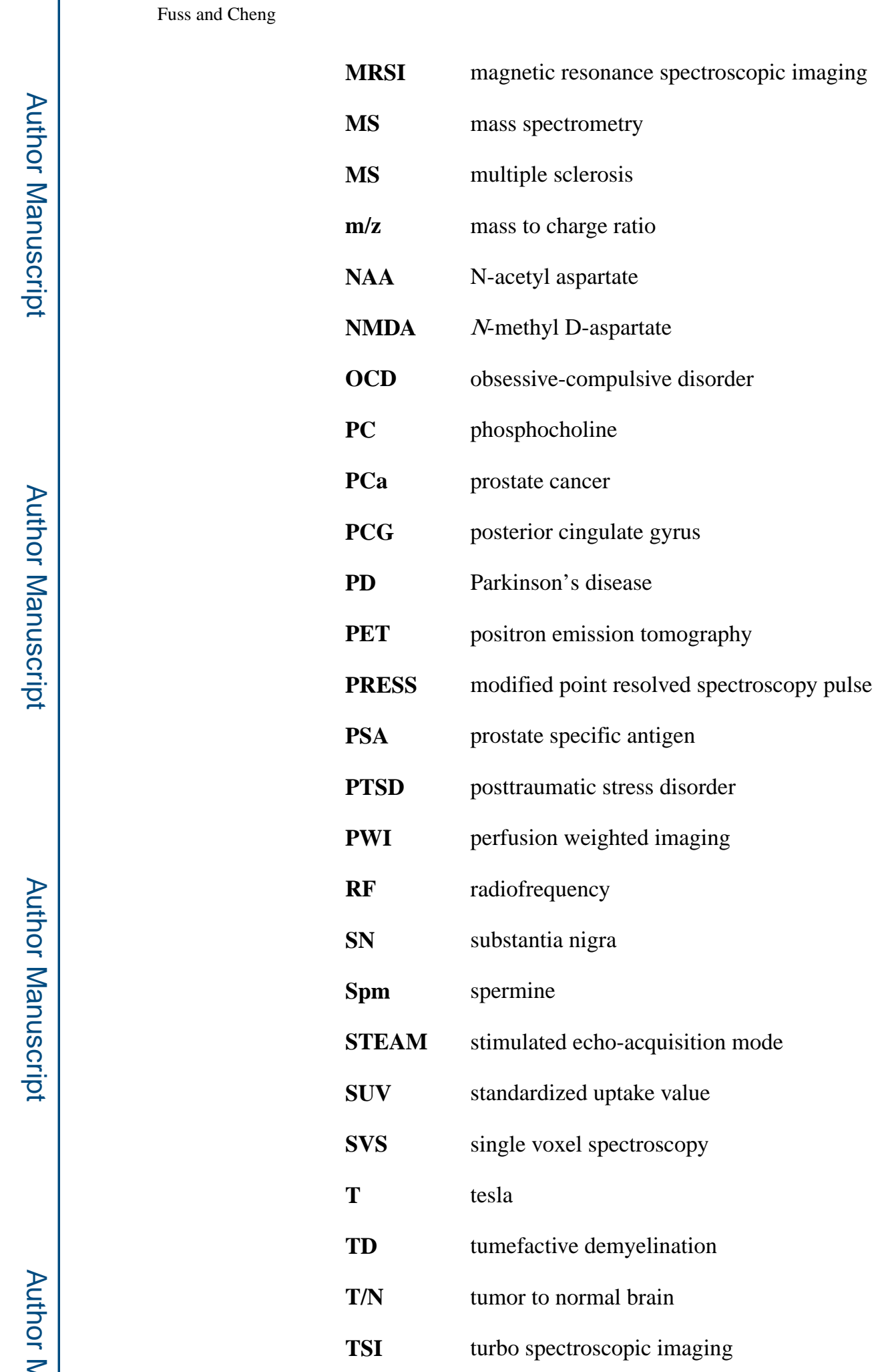

References

1. Kudo T. Metabolic imaging using PET. European journal of nuclear medicine and molecular imaging. 2007 Jun; 34(Suppl 1):S49-S61. [PubMed: 17486339]

Top Magn Reson Imaging. Author manuscript; available in PMC 2017 October 01. 
2. Horska A, Barker PB. Imaging of brain tumors: MR spectroscopy and metabolic imaging. Neuroimaging clinics of North America. 2010 Aug; 20(3):293-310. [PubMed: 20708548]

3. Haga KK, Khor YP, Farrall A, Wardlaw JM. A systematic review of brain metabolite changes, measured with $1 \mathrm{H}$ magnetic resonance spectroscopy, in healthy aging. Neurobiology of aging. 2009 Mar; 30(3):353-363. [PubMed: 17719145]

4. Saunders DE, Howe FA, van den Boogaart A, Griffiths JR, Brown MM. Aging of the adult human brain: in vivo quantitation of metabolite content with proton magnetic resonance spectroscopy. Journal of magnetic resonance imaging : JMRI. 1999 May; 9(5):711-716. [PubMed: 10331768]

5. Chang L, Ernst T, Poland RE, Jenden DJ. In vivo proton magnetic resonance spectroscopy of the normal aging human brain. Life sciences. 1996; 58(22):2049-2056. [PubMed: 8637436]

6. Yang A, Xiao X, Wang Z. Evaluation of normal changes in pons metabolites due to aging using turbo spectroscopic imaging. AJNR. American journal of neuroradiology. 2014 Nov-Dec;35(11): 2099-2105. [PubMed: 24994832]

7. Gruber S, Bogner W, Stadlbauer A, Krssak M, Bodamer O. Magnetic resonance spectroscopy in patients with Fabry and Gaucher disease. European journal of radiology. 2011 Aug; 79(2):295-298. [PubMed: 20227208]

8. Levin BE, Katzen HL, Maudsley A, et al. Whole-brain proton MR spectroscopic imaging in Parkinson's disease. Journal of neuroimaging : official journal of the American Society of Neuroimaging. 2014 Jan-Feb;24(1):39-44. [PubMed: 23228009]

9. Groger A, Bender B, Wurster I, Chadzynski GL, Klose U, Berg D. Differentiation between idiopathic and atypical parkinsonian syndromes using three-dimensional magnetic resonance spectroscopic imaging. Journal of neurology, neurosurgery, and psychiatry. 2013 Jun; 84(6):644649.

10. Zhong X, Shi H, Shen Z, et al. 1H-proton magnetic resonance spectroscopy differentiates dementia with Lewy bodies from Alzheimer's disease. Journal o Alzheimer's disease : JAD. 2014; 40(4): 953-966.

11. Wang Y, Li D, Bao F, et al. Thalamic metabolic alterations with cognitive dysfunction in idiopathic trigeminal neuralgia: a multivoxel spectroscopy study. Neuroradiology. 2014 Aug; 56(8):685-693. [PubMed: 24820951]

12. Govind V, Sharma KR, Maudsley AA, Arheart KL, Saigal G, Sheriff S. Comprehensive evaluation of corticospinal tract metabolites in amyotrophic lateral sclerosis using whole-brain $1 \mathrm{H} \mathrm{MR}$ spectroscopy. PloS one. 2012; 7(4):e35607. [PubMed: 22539984]

13. van den Bogaard SJ, Dumas EM, Teeuwisse WM, et al. Exploratory 7-Tesla magnetic resonance spectroscopy in Huntington's disease provides in vivo evidence for impaired energy metabolism. Journal of neurology. 2011 Dec; 258(12):2230-2239. [PubMed: 21614431]

14. Tur C, Wheeler-Kingshott CA, Altmann DR, Miller DH, Thompson AJ, Ciccarelli O. Spatial variability and changes of metabolite concentrations in the cortico-spinal tract in multiple sclerosis using coronal CSI. Human brain mapping. 2014 Mar; 35(3):993-1003. [PubMed: 23281189]

15. Saini J, Chatterjee S, Thomas B, Kesavadas C. Conventional and advanced magnetic resonance imaging in tumefactive demyelination. Acta radiologica (Stockholm, Sweden: 1987). 2011 Dec 1; 52(10):1159-1168.

16. Meyerhoff DJ, Mon A, Metzler T, Neylan TC. Cortical gamma-aminobutyric acid and glutamate in posttraumatic stress disorder and their relationships to self-reported sleep quality. Sleep. 2014 May; 37(5):893-900. [PubMed: 24790267]

17. Pennington DL, Abe C, Batki SL, Meyerhoff DJ. A preliminary examination of cortical neurotransmitter levels associated with heavy drinking in posttraumatic stress disorder. Psychiatry research. 2014 Dec 30; 224(3):281-287. [PubMed: 25444536]

18. Tandon N, Bolo NR, Sanghavi K, et al. Brain metabolite alterations in young adults at familial high risk for schizophrenia using proton magnetic resonance spectroscopy. Schizophrenia research. 2013 Aug; 148(1-3):59-66. [PubMed: 23791389]

19. Howells FM, Ives-Deliperi VL, Horn NR, Stein DJ. Increased thalamic phospholipid concentration evident in bipolar I disorder. Progress in neuro-psychopharmacology \& biological psychiatry. 2013 Mar 5.41:1-5. [PubMed: 23142769]

Top Magn Reson Imaging. Author manuscript; available in PMC 2017 October 01. 
20. Xu J, Dydak U, Harezlak J, et al. Neurochemical abnormalities in unmedicated bipolar depression and mania: a 2D 1H MRS investigation. Psychiatry research. 2013 Sep 30; 213(3):235-241. [PubMed: 23810639]

21. Howells FM, Uhlmann A, Temmingh H, et al. (1)H-magnetic resonance spectroscopy ((1)H-MRS) in methamphetamine dependence and methamphetamine induced psychosis. Schizophrenia research. 2014 Mar; 153(1-3):122-128. [PubMed: 24529366]

22. Jia Y, Zhong S, Wang Y, Liu T, Liao X, Huang L. The correlation between biochemical abnormalities in frontal white matter, hippocampus and serum thyroid hormone levels in firstepisode patients with major depressive disorder. Journal of affective disorders. $2015 \mathrm{Jul}$ 15.180:162-169. [PubMed: 25913802]

23. O'Neill J, Piacentini JC, Chang S, et al. MRSI correlates of cognitive-behavioral therapy in pediatric obsessive-compulsive disorder. Progress in neuro-psychopharmacology \& biological psychiatry. 2012 Jan 10; 36(1):161-168. [PubMed: 21983143]

24. Yang ZX, Huo SS, Cheng XF, et al. Quantitative multivoxel proton MR spectroscopy study of brain metabolites in patients with amnestic mild cognitive impairment: a pilot study. Neuroradiology. 2012 May; 54(5):451-458. [PubMed: 21739221]

25. Zimny A, Bladowska J, Macioszek A, et al. Evaluation of the posterior cingulate region with FDGPET and advanced MR techniques in patients with amnestic mild cognitive impairment: comparison of the methods. Journal of Alzheimer's disease : JAD. 2015; 44(1):329-338.

26. Alkan A, Sharifov R, Akkoyunlu ME, et al. MR spectroscopy features of brain in patients with mild and severe obstructive sleep apnea syndrome. Clinical imaging. 2013 Nov-Dec;37(6):989992. [PubMed: 23993754]

27. Ristic AJ, Ostojic J, Kozic D, et al. Hippocampal metabolic dysfunction in juvenile myoclonic epilepsy: 3D multivoxel spectroscopy study. Journal of the neurological sciences. 2011 Jun 15; 305(1-2):139-142. [PubMed: 21420103]

28. Cecil KM, Dietrich KN, Altaye M, et al. Proton magnetic resonance spectroscopy in adults with childhood lead exposure. Environmental health perspectives. 2011 Mar; 119(3):403-408. [PubMed: 20947467]

29. Li J, Zhang XY, Wang B, et al. Multivoxel proton magnetic resonance spectroscopy in heat stroke. Clinical radiology. 2015 Jan; 70(1):37-41. [PubMed: 25443777]

30. Ferlay J, Soerjomataram I, Dikshit R, et al. Cancer incidence and mortality worldwide: Sources, methods and major patterns in GLOBOCAN 2012. International Journal of Cancer. 2015; 136(5):E359-E386. [PubMed: 25220842]

31. Caivano R, Lotumolo A, Rabasco P, et al. 3 Tesla magnetic resonance spectroscopy: cerebral gliomas vs. metastatic brain tumors. Our experience and review of the literature. The International journal of neuroscience. 2013 Aug; 123(8):537-543. [PubMed: 23390934]

32. Georgiadis P, Kostopoulos S, Cavouras D, et al. Quantitative combination of volumetric MR imaging and MR spectroscopy data for the discrimination of meningiomas from metastatic brain tumors by means of pattern recognition. Magnetic resonance imaging. 2011 May; 29(4):525-535. [PubMed: 21315534]

33. Andronesi OC, Kim GS, Gerstner E, et al. Detection of 2-hydroxyglutarate in IDH-mutated glioma patients by in vivo spectral-editing and 2D correlation magnetic resonance spectroscopy. Science translational medicine. 2012 Jan 11.4(116):116ra114.

34. Santagata S, Eberlin LS, Norton I, et al. Intraoperative mass spectrometry mapping of an oncometabolite to guide brain tumor surgery. Proceedings of the National Academy of Sciences of the United States of America. 2014 Jul 29; 111(30):11121-11126. [PubMed: 24982150]

35. Chen WS, Li JJ, Zhang JH, et al. Magnetic resonance spectroscopic imaging of brain injury after nasopharyngeal cancer radiation in early delayed reaction. Genetics and molecular research : GMR. 2014; 13(3):6848-6854. [PubMed: 25177964]

36. Steffen-Smith EA, Venzon DJ, Bent RS, Hipp SJ, Warren KE. Single- and multivoxel proton spectroscopy in pediatric patients with diffuse intrinsic pontine glioma. International journal of radiation oncology, biology, physics. 2012 Nov 1; 84(3):774-779.

Top Magn Reson Imaging. Author manuscript; available in PMC 2017 October 01. 
37. Bieza A, Krumina G. Magnetic resonance study on fractional anisotropy and neuronal metabolite ratios in peritumoral area of cerebral gliomas. Medicina (Kaunas, Lithuania). 2012; 48(10):497506.

38. Bisdas S, Ritz R, Bender B, et al. Metabolic mapping of gliomas using hybrid MR-PET imaging: feasibility of the method and spatial distribution of metabolic changes. Investigative radiology. 2013 May; 48(5):295-301. [PubMed: 23296081]

39. van Dorsten FA, van der Graaf M, Engelbrecht MR, et al. Combined quantitative dynamic contrastenhanced MR imaging and (1)H MR spectroscopic imaging of human prostate cancer. Journal of magnetic resonance imaging : JMRI. 2004 Aug; 20(2):279-287. [PubMed: 15269954]

40. Cunningham CH, Vigneron DB, Marjanska M, et al. Sequence design for magnetic resonance spectroscopic imaging of prostate cancer at 3 T. Magnetic resonance in medicine. 2005 May; 53(5):1033-1039. [PubMed: 15844147]

41. Coakley FV, Qayyum A, Kurhanewicz J. Magnetic resonance imaging and spectroscopic imaging of prostate cancer. The Journal of urology. 2003 Dec; $170(6$ Pt 2):S69-S75. discussion S75-66. [PubMed: 14610414]

42. Carlani M, Mancino S, Bonanno E, Finazzi Agro E, Simonetti G. Combined morphological, [1H]MR spectroscopic and contrast-enhanced imaging of human prostate cancer with a 3-Tesla scanner: preliminary experience. La Radiologia medica. 2008 Aug; 113(5):670-688. [PubMed: 18493829]

43. Swanson MG, Vigneron DB, Tabatabai ZL, et al. Proton HR-MAS spectroscopy and quantitative pathologic analysis of MRI/3D-MRSI-targeted postsurgical prostate tissues. Magnetic resonance in medicine. 2003 Nov; 50(5):944-954. [PubMed: 14587005]

44. Mueller-Lisse UG, Swanson MG, Vigneron DB, et al. Time-dependent effects of hormonedeprivation therapy on prostate metabolism as detected by combined magnetic resonance imaging and 3D magnetic resonance spectroscopic imaging. Magnetic resonance in medicine. $2001 \mathrm{Jul}$; 46(1):49-57. [PubMed: 11443710]

45. Pickett B, Kurhanewicz J, Coakley F, Shinohara K, Fein B, Roach M 3rd. Use of MRI and spectroscopy in evaluation of external beam radiotherapy for prostate cancer. International journal of radiation oncology, biology, physics. 2004 Nov 15; 60(4):1047-1055.

46. Pickett B, Ten Haken RK, Kurhanewicz J, et al. Time to metabolic atrophy after permanent prostate seed implantation based on magnetic resonance spectroscopic imaging. International journal of radiation oncology, biology, physics. 2004 Jul 1; 59(3):665-673.

47. Giusti S, Caramella D, Fruzzetti E, Lazzereschi M, Tognetti A, Bartolozzi C. Peripheral zone prostate cancer. Pre-treatment evaluation with MR and 3D (1)H MR spectroscopic imaging: correlation with pathologic findings. Abdominal imaging. 2010 Dec; 35(6):757-763. [PubMed: 19756851]

48. Kobus T, Hambrock T, Hulsbergen-van de Kaa CA, et al. In vivo assessment of prostate cancer aggressiveness using magnetic resonance spectroscopic imaging at $3 \mathrm{~T}$ with an endorectal coil. European urology. 2011 Nov; 60(5):1074-1080. [PubMed: 21419565]

49. Selnaes KM, Gribbestad IS, Bertilsson H, et al. Spatially matched in vivo and ex vivo MR metabolic profiles of prostate cancer -- investigation of a correlation with Gleason score. NMR in biomedicine. 2013 May; 26(5):600-606. [PubMed: 23280546]

50. Sciarra A, Panebianco V, Salciccia S, et al. Determination of the time for maximal response to neoadjuvant hormone therapy for prostate cancer using magnetic resonance with spectroscopy (MRSI) and dynamic contrast enhancement (DCEMR). Urologic oncology. 2012 Sep; 30(5):614619. [PubMed: 21396849]

51. Valentini AL, Gui B, D'Agostino GR, et al. Locally advanced prostate cancer: three-dimensional magnetic resonance spectroscopy to monitor prostate response to therapy. International journal of radiation oncology, biology, physics. 2012 Nov 1; 84(3):719-724.

52. Panebianco V, Barchetti F, Musio D, et al. Metabolic atrophy and 3-T 1H-magnetic resonance spectroscopy correlation after radiation therapy for prostate cancer. BJU international. 2014 Dec; 114(6):852-859. [PubMed: 24180518]

53. Kirilova A, Damyanovich A, Crook J, Jezioranski J, Wallace K, Pintilie M. 3D MR-spectroscopic imaging assessment of metabolic activity in the prostate during the PSA "bounce" following

Top Magn Reson Imaging. Author manuscript; available in PMC 2017 October 01. 
125iodine brachytherapy. International journal of radiation oncology, biology, physics. 2011 Feb 1; 79(2):371-378.

54. Venugopal N, McCurdy B, Al Mehairi S, et al. Short echo time in vivo prostate (1)H-MRSI. Magnetic resonance imaging. 2012 Feb; 30(2):195-204. [PubMed: 22154684]

55. Nelson SJ, Kurhanewicz J, Vigneron DB, et al. Metabolic imaging of patients with prostate cancer using hyperpolarized [1-(1)(3)C]pyruvate. Science translational medicine. 2013 Aug 14.5(198): 198ra108.

56. Dou W, Palomero-Gallagher N, van Tol MJ, et al. Systematic regional variations of GABA, glutamine, and glutamate concentrations follow receptor fingerprints of human cingulate cortex. The Journal of neuroscience : the official journal of the Society for Neuroscience. $2013 \mathrm{Jul}$ 31; 33(31):12698-12704. [PubMed: 23904606]

57. Ganji SK, An Z, Banerjee A, Madan A, Hulsey KM, Choi C. Measurement of regional variation of GABA in the human brain by optimized point-resolved spectroscopy at $7 \mathrm{~T}$ in vivo. NMR in biomedicine. 2014 Oct; 27(10):1167-1175. [PubMed: 25088346]

58. Tal A, Kirov II, Grossman RI, Gonen O. The role of gray and white matter segmentation in quantitative proton MR spectroscopic imaging. NMR in biomedicine. 2012 Dec; 25(12):13921400. [PubMed: 22714729]

59. Bednarik P, Tkac I, Giove F, et al. Neurochemical and BOLD responses during neuronal activation measured in the human visual cortex at 7 Tesla. Journal of cerebral blood flow and metabolism : official journal of the International Society of Cerebral Blood Flow and Metabolism. 2015 Apr; 35(4):601-610.

60. Wu CL, Jordan KW, Ratai EM, et al. Metabolomic imaging for human prostate cancer detection. Science translational medicine. 2010 Jan 27.2(16):16ra18.

61. Cheng LL, Lean CL, Bogdanova A, et al. Enhanced resolution of proton NMR spectra of malignant lymph nodes using magic-angle spinning. Magnetic resonance in medicine. 1996 Nov; 36(5):653-658. [PubMed: 8916014] 
Controls
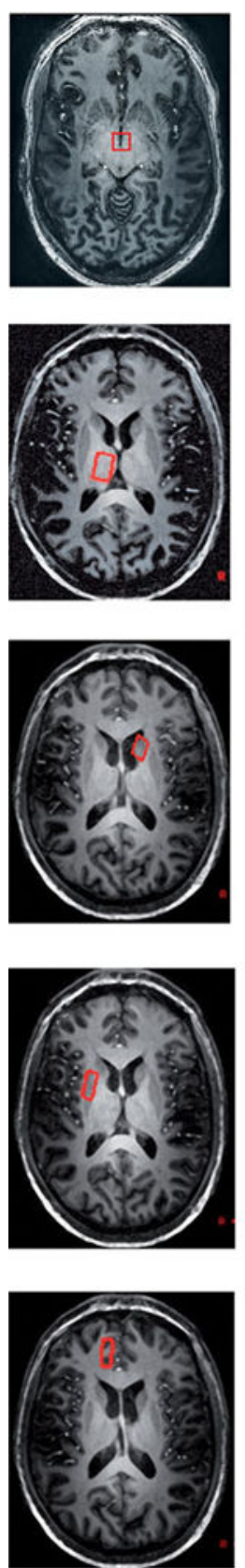

\section{Hypothalamus}

\section{Thalamus}

\section{Putamen}

Prefrontal
Premanifest

Manifest
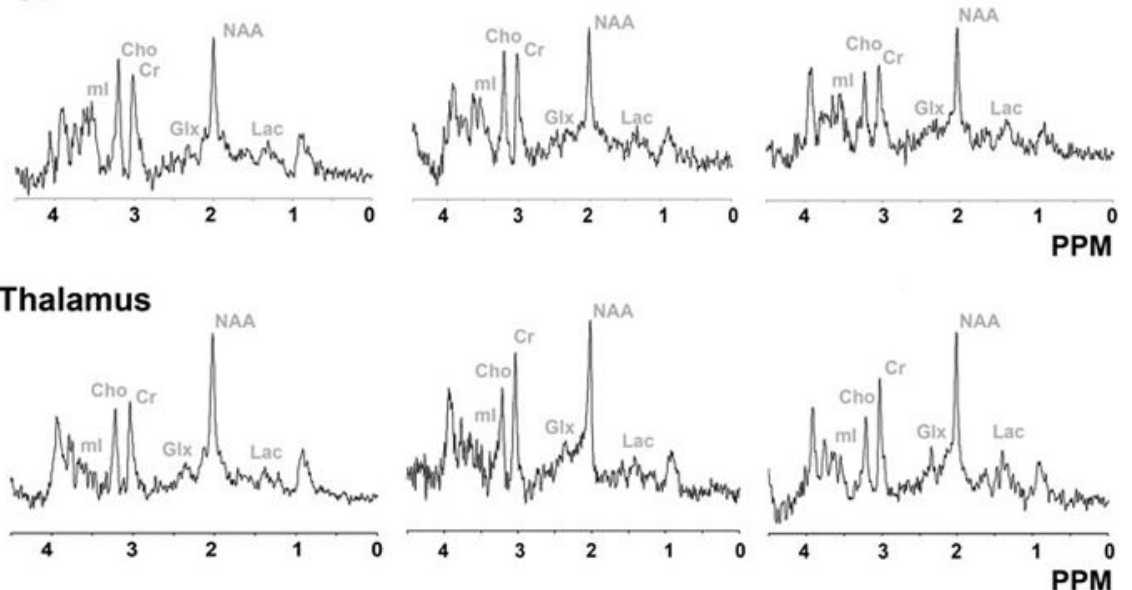

\section{Caudate Nucleus}
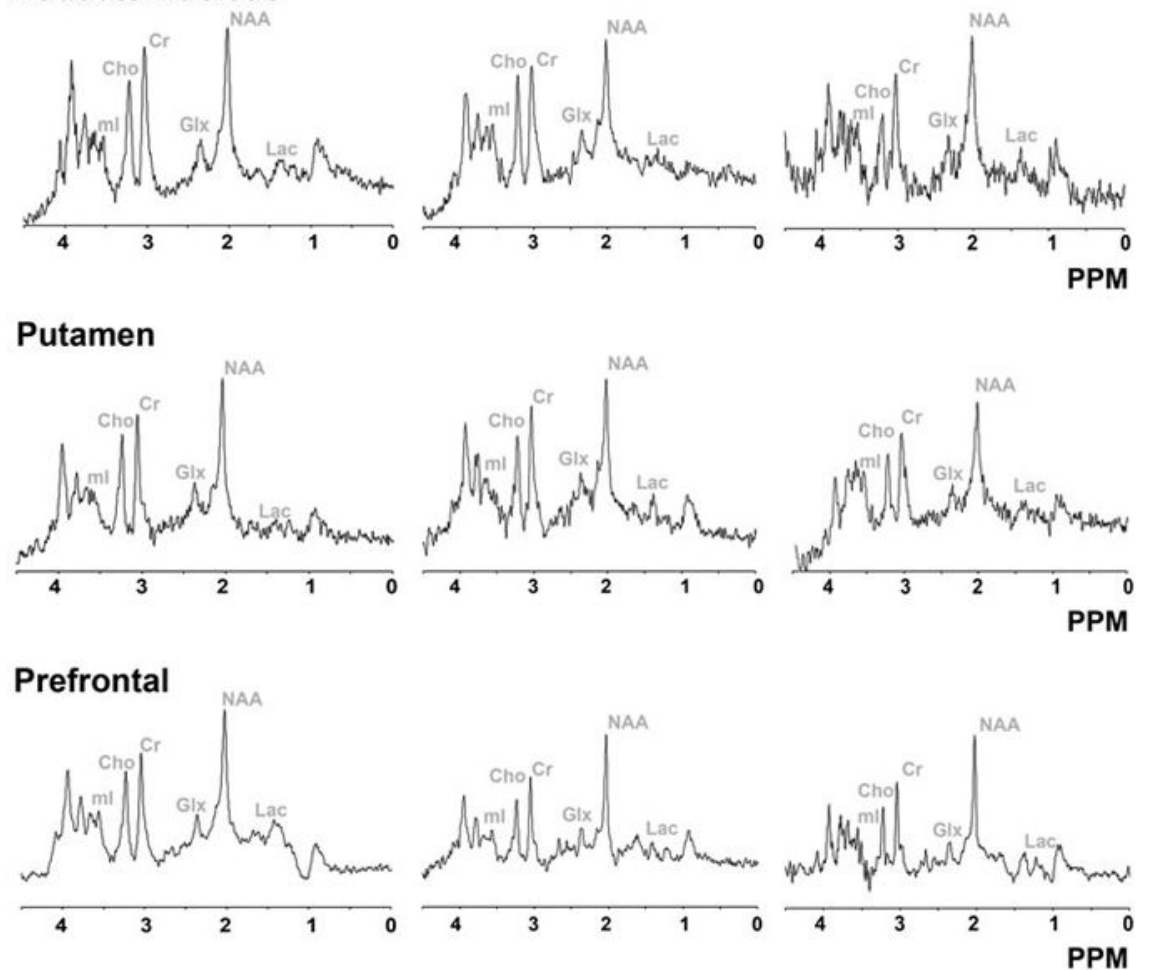

Figure 1.

Localized proton MR spectra from different regions of the brain. On the left side, the voxel is displayed in the transverse direction, a typical spectrum of that structure is shown on the right side for the three groups. Five different regions are displayed: hypothalamus, thalamus, caudate nucleus, putamen, prefrontal region. A Gaussian filter of $4 \mathrm{~Hz}$ was applied ${ }^{13}$. 

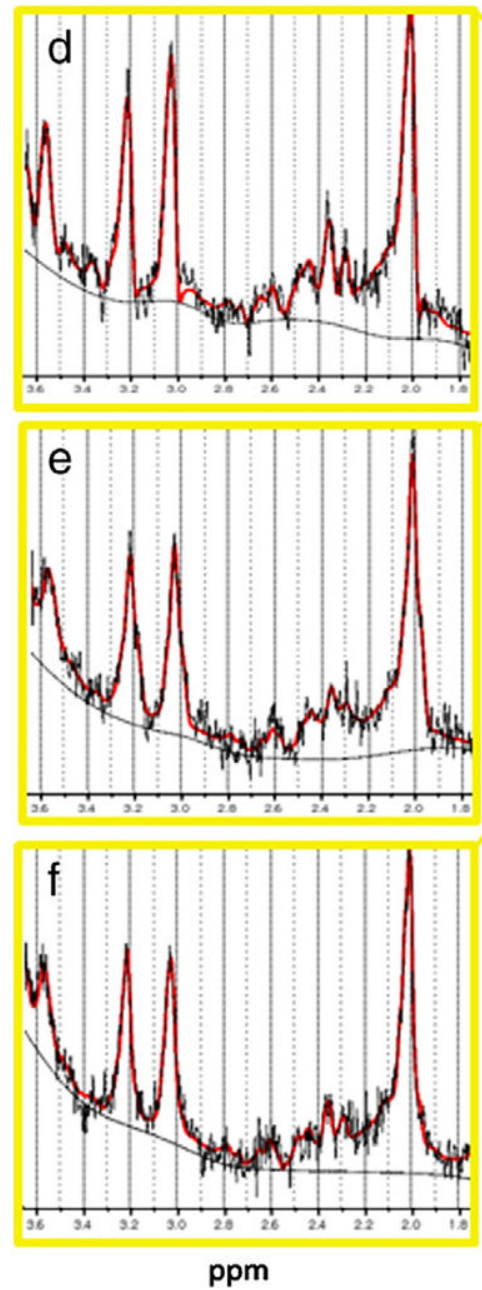

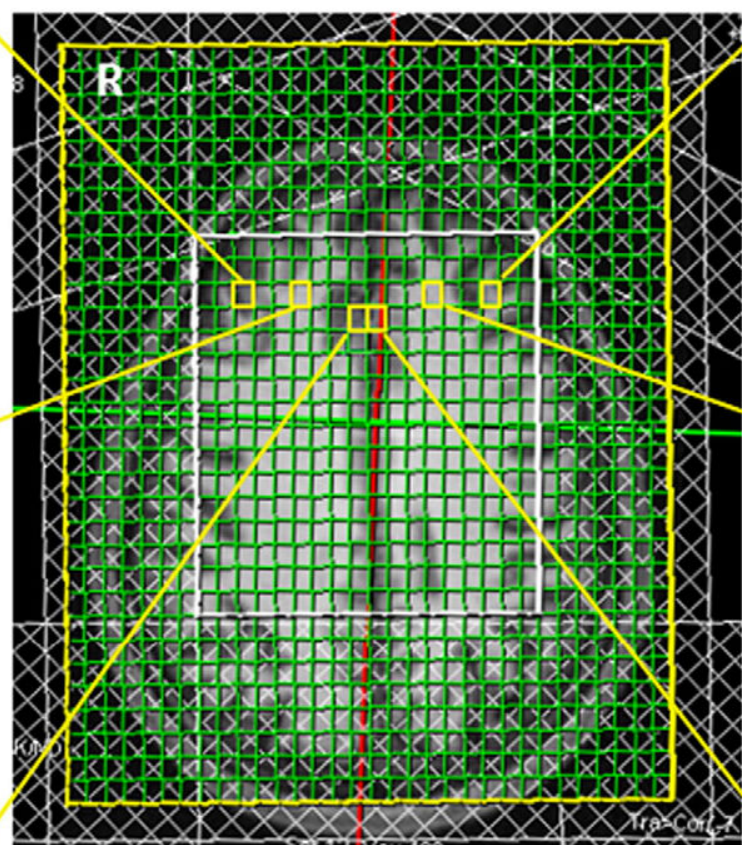

a

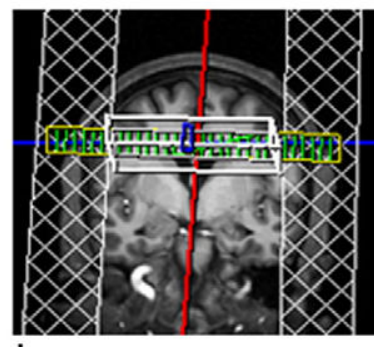

b

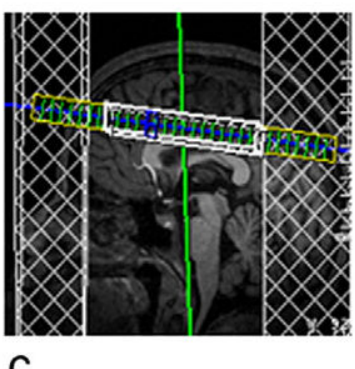

C
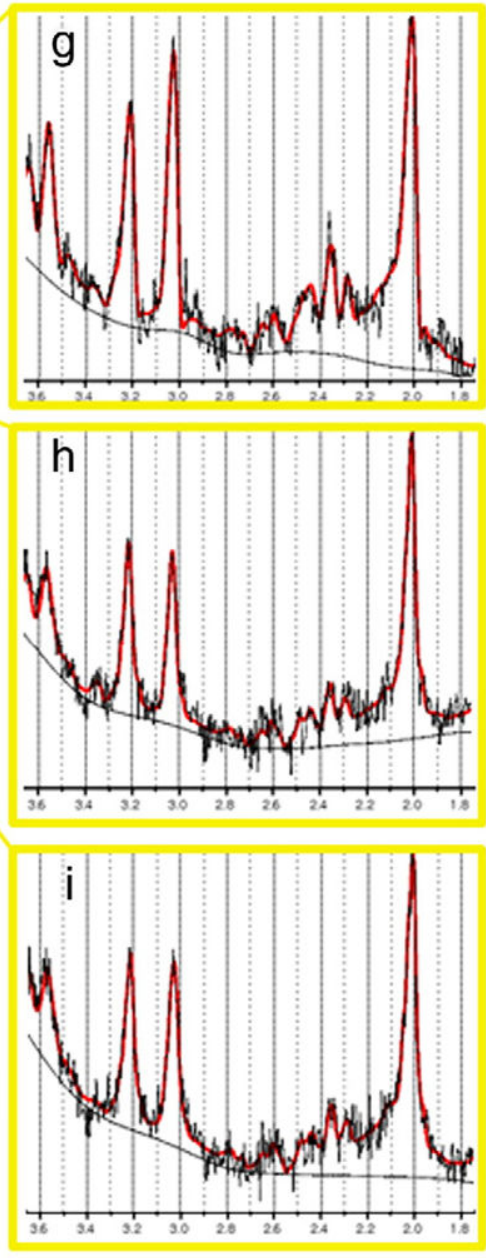

ppm

Figure 2.

Representative 1H-MRS 2D chemical shift imaging brain slice orientation on the 3D planes ( $\mathrm{a}$ - axial, b - coronal, c - sagittal) and spectra of VOIswhich included: (d \& g) bilateral anterior cingulate cortices (ACC), (f \& i) bilateral dorsolateral prefrontal cortices (DLPFC), and $\left(\mathrm{e} \& \mathrm{~h}\right.$ ) bilateral frontal white matter voxels located between ACC and DLFPC ${ }^{21}$. 


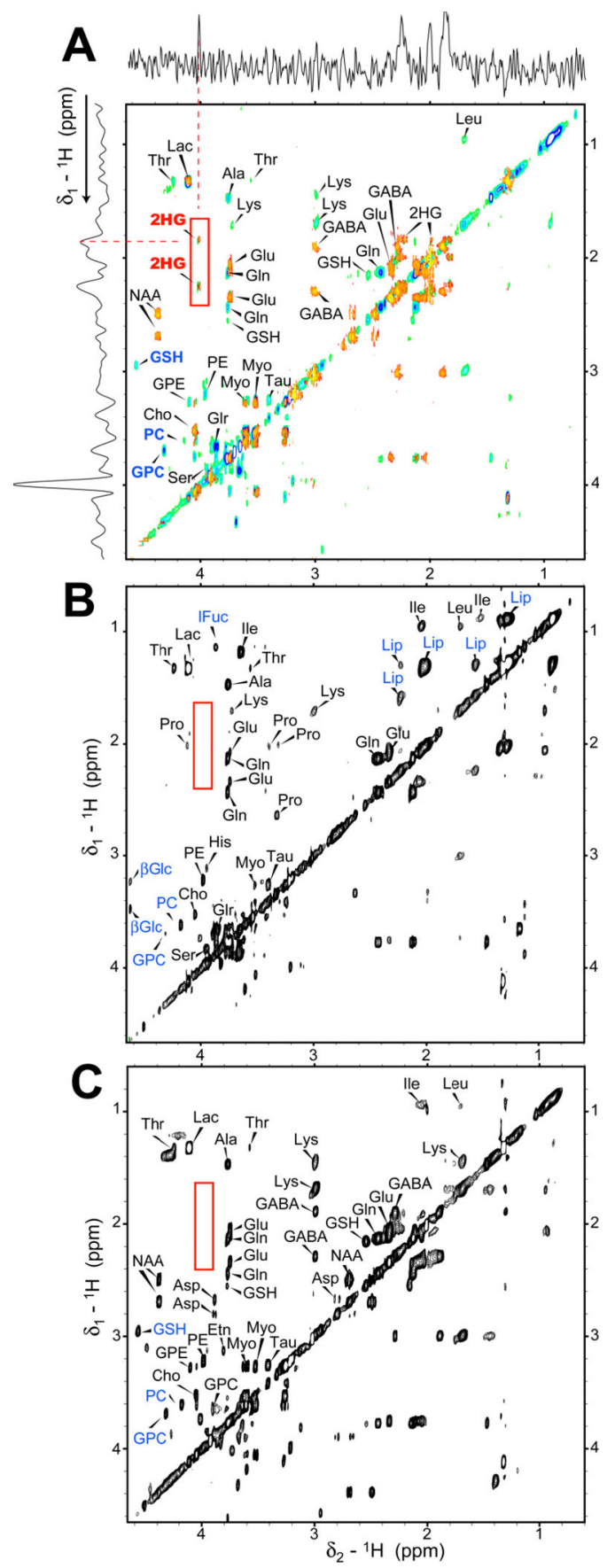

Figure 3.

HR-MAS spectra recorded at $14 \mathrm{~T}$ ex vivo on biopsy tissue from patients with and without IDH1 mutations. 1H-1H 2D TOBSY spectra are shown for all biopsies (the minimum contour levels were set five times the noise level). (A) For anaplastic astrocytoma biopsy tissue with IDH1R132 mutation $(n=1)$, the spectra are shown in green-blue contours. The phantom is shown in red-yellow. Projections along $\delta 1$ and $\delta 2$ show the $2 \mathrm{HG}$ crosspeaks, outlined by a red rectangle. (B and $\mathbf{C}$ ) Spectra for wt-IDH1 patients: primary glioblastoma $(B ; n=1)$ and non-tumor $(C ; n=1)$. The region where $2 \mathrm{HG}$ crosspeaks would be expected is 
outlined by a red rectangle. For all 2D TOBSY brain spectra, several other metabolites can be identified. Amino acids: alanine (Ala), aspartate (Asp), glutamate (Glu), glutamine (Gln), histidine (His), isoleucine (Ile), leucine (Leu), lysine (Lys), proline (Pro), serine (Ser), threonine (Thr). Membrane phospholipid-related compounds: choline (Cho), ethanolamine (Etn), glycerol (Glr), glycerophosphocholine (GPC), glycerophosphoethanolamine (GPE), phosphocholine (PC), phosphoethanolamine (PE). Sugars: l-fucose (lFuc), $\beta$-glucose (bGlc), myo-inositol (Myo). Miscellaneous: glutathione (GSH), lactate (Lac), lipids (Lip), Nacetylaspartate (NAA), and taurine (Tau ${ }^{33}$. 
A Diagnostic

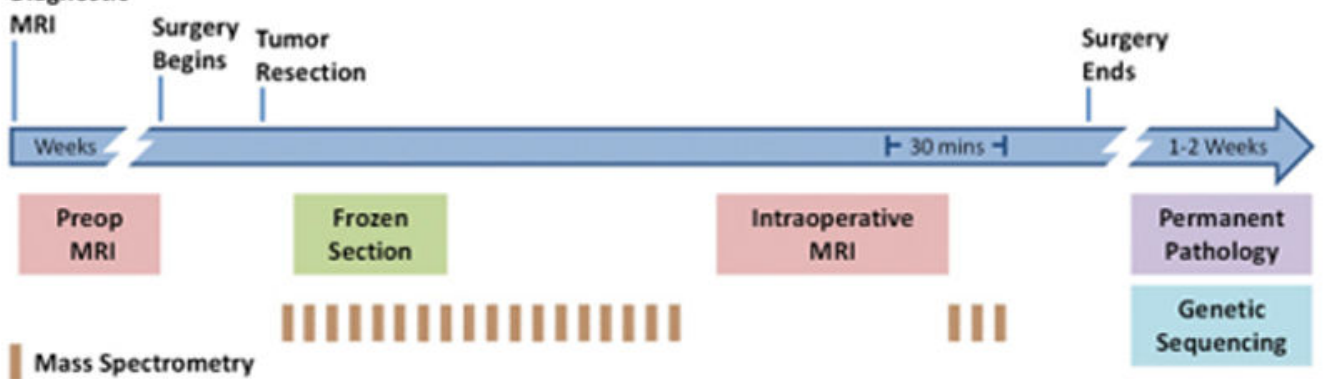

B S72
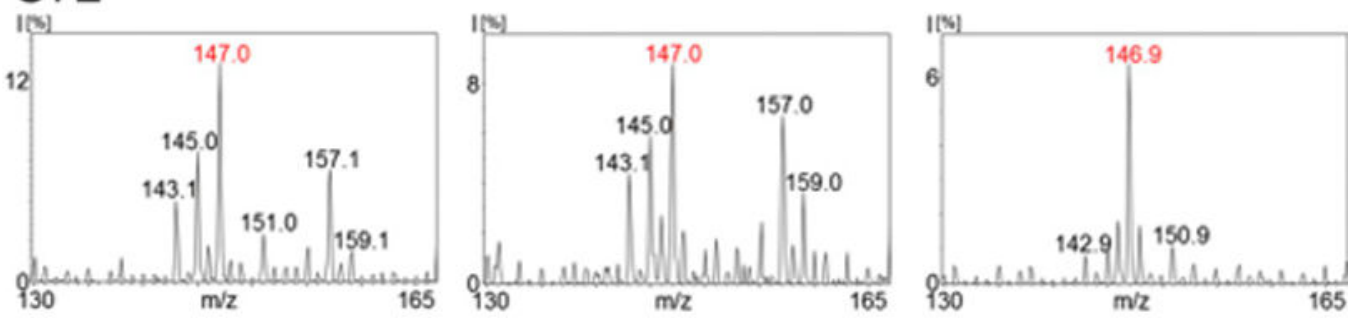

C
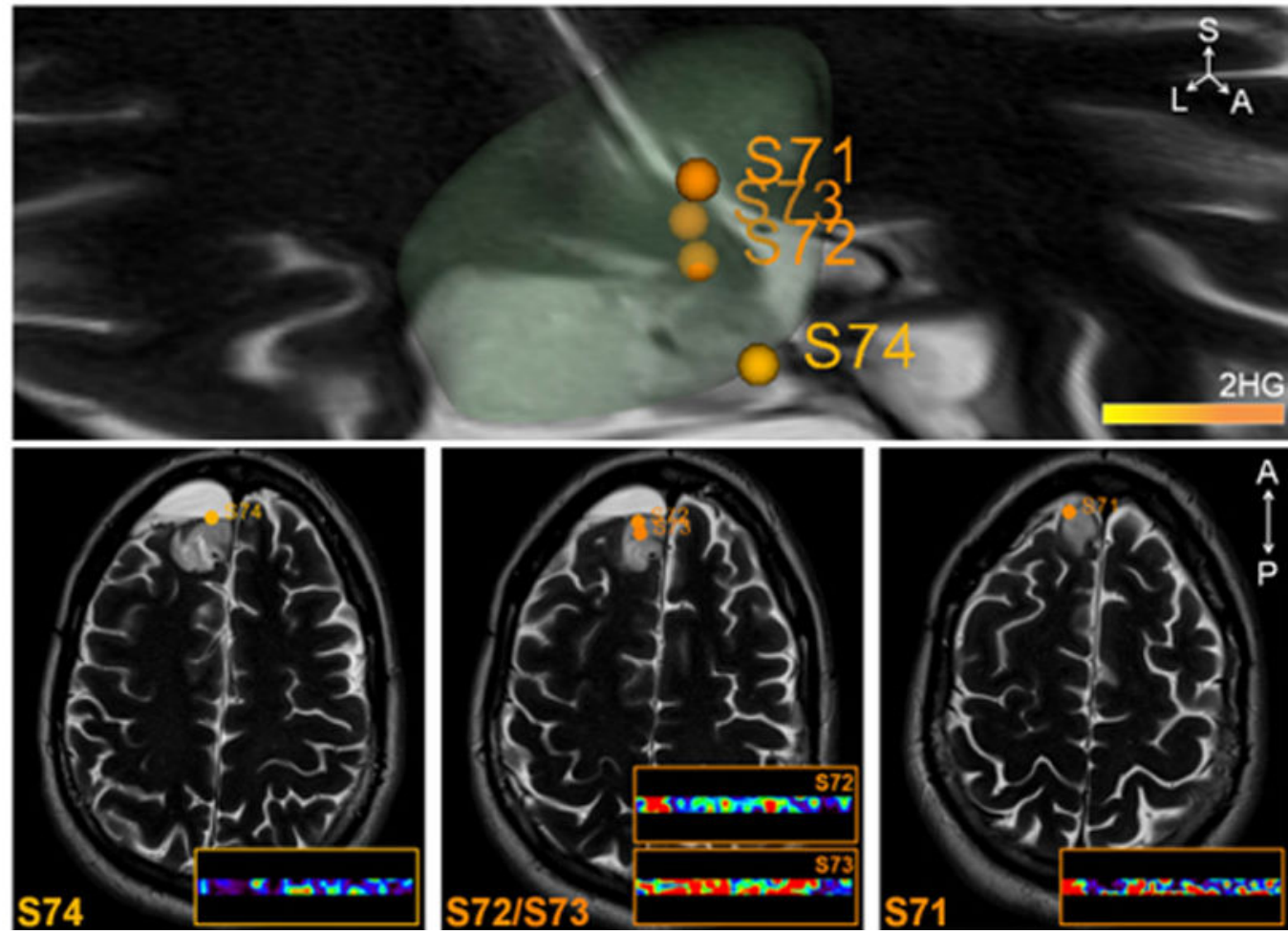

Figure 4.

(A) Time course and work flow of patient care associated with a typical 5-h neurosurgery in the AMIGO, MRI-equipped, operative suite at Brigham and Women's Hospital. (B)

Negative ion mode DESI mass spectra obtained using an amaZon Speed ion trap from $\mathrm{m} / \mathrm{z}$ 130-165 (Bruker Daltonics) from a swab (Left), a smear (Center), and a section (Right) for sample S72. (C) Normalized 2-HG signal is represented with a warm color scale as indicated by the scale bar, set from the lowest (yellow) to highest (orange) levels detected from this individual case. Stereotactic positions were digitally registered to the preoperative MRI 
using neuronavigation (BrainLab system) in a standard operating room. The 3D tumor volume is shown (Upper). Classification results of samples S74, S72, S73, and S71 are further visualized on axial sections (Lower) ${ }^{34}$. 


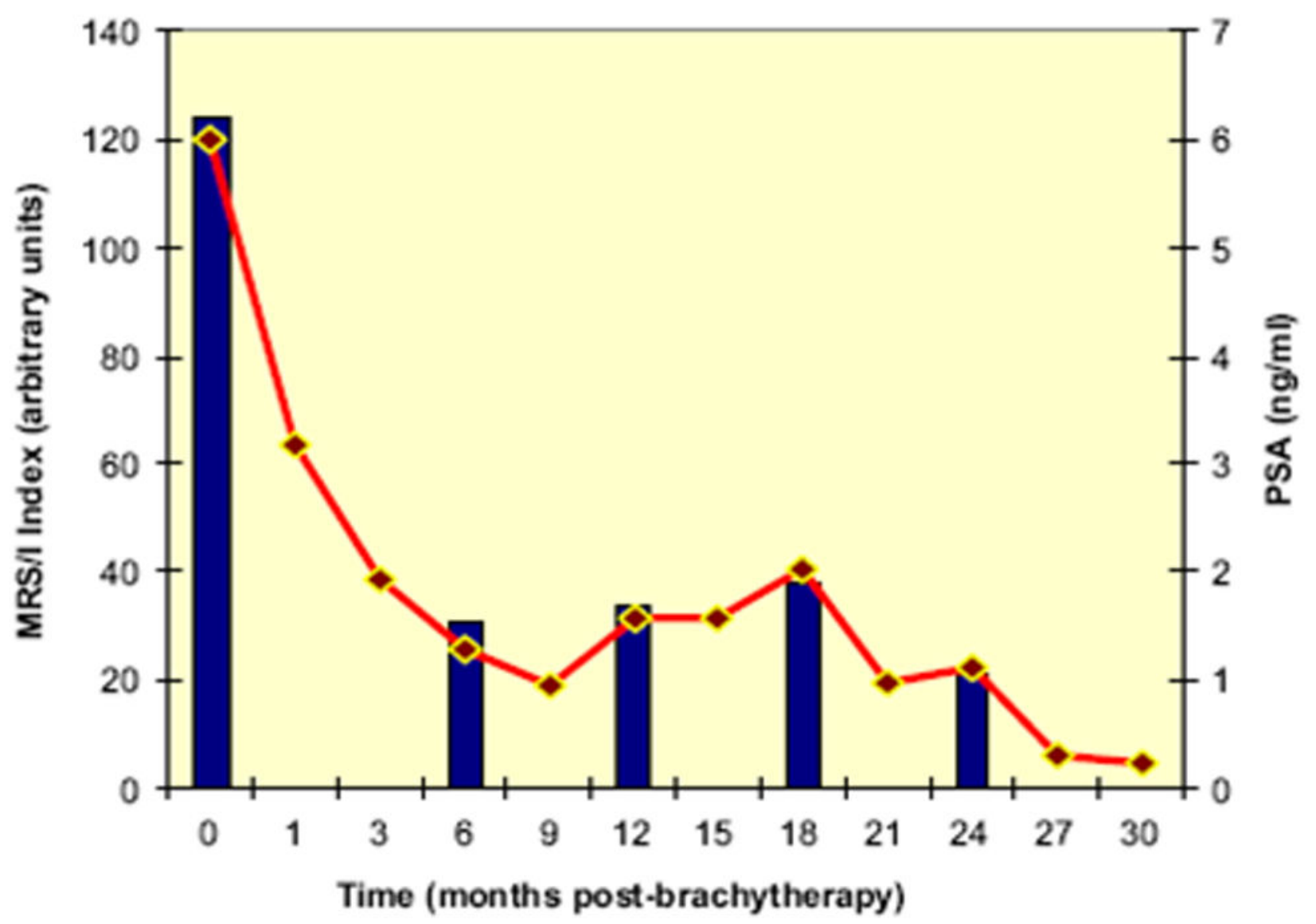

Figure 5.

Example of the relationship between prostate-specific antigen (PSA) and metabolic activity during a PSA bounce for one patient. Magnetic resonance spectroscopy (MRSI) index is $*[\mathrm{Cho}+\mathrm{Cr}] / \mathrm{Cit}$ _ (signal/noise $)_{\text {choline }}{ }^{53}$. 

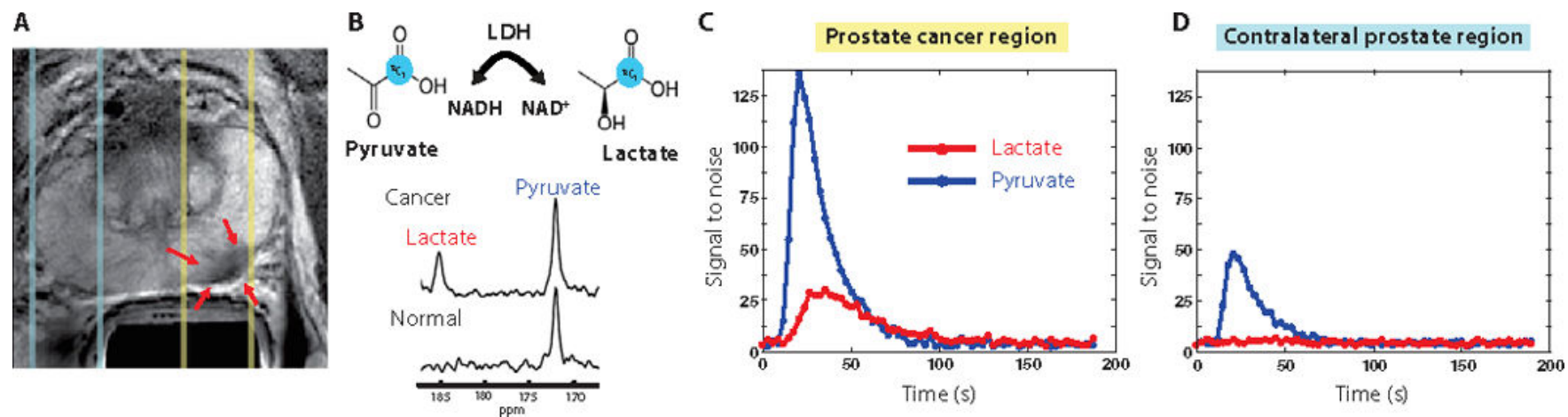

Figure 6.

Images are from a representative patient with a current PSA od $12.2 \mathrm{ng} / \mathrm{ml}$, a small volume of biopsy-proven Gleason grade $4+3 \mathrm{PCa}$ in the left midgland, and who received the lowest dose $(0.14 \mathrm{ml} / \mathrm{kg})$ of hyperpolarized $\left[1-{ }^{13} \mathrm{C}\right]$ pyruvate. (A) Axial $\mathrm{T}_{2}$-weighted image showing slices (dashed lines) obtained from 1D spectral localization. The slice that overlaps the left prostatic peropheral zone (right side of image) contained a small focus of reduced $\mathrm{T}_{2}$ signal intensity corresponding to the region of biopsy-proven PCa (red arrows). The slice overlapping the right peripheral zone (left side of image) contains only normal prostate tissue. (B) Flux of $\left[1-{ }^{13} \mathrm{C}\right]$ pyruvate to $\left[1-{ }^{13} \mathrm{C}\right]$ lactate catalyzed by LDH (top) Dynamic ${ }^{13} \mathrm{C}$ spectra were obtained from the same patient in (A) at $36 \mathrm{~s}$ after injection of hyperpolarized $\left[1-{ }^{13} \mathrm{C}\right]$ pyruvate (bottom). The cancer spectrum demonstrated a lactate SNR of 25 owing to the high flux of hyperpolarized $\left[1-{ }^{13} \mathrm{C}\right]$ pyruvate to $\left[1-{ }^{13} \mathrm{C}\right]$ lactate. $(\mathrm{C})$ Plot of $1 \mathrm{D}$ localized dynamic hyperpolarized pyruvate and lactate data from the slice that overlapped a contralateral region of the prostate ${ }^{55}$. 
A

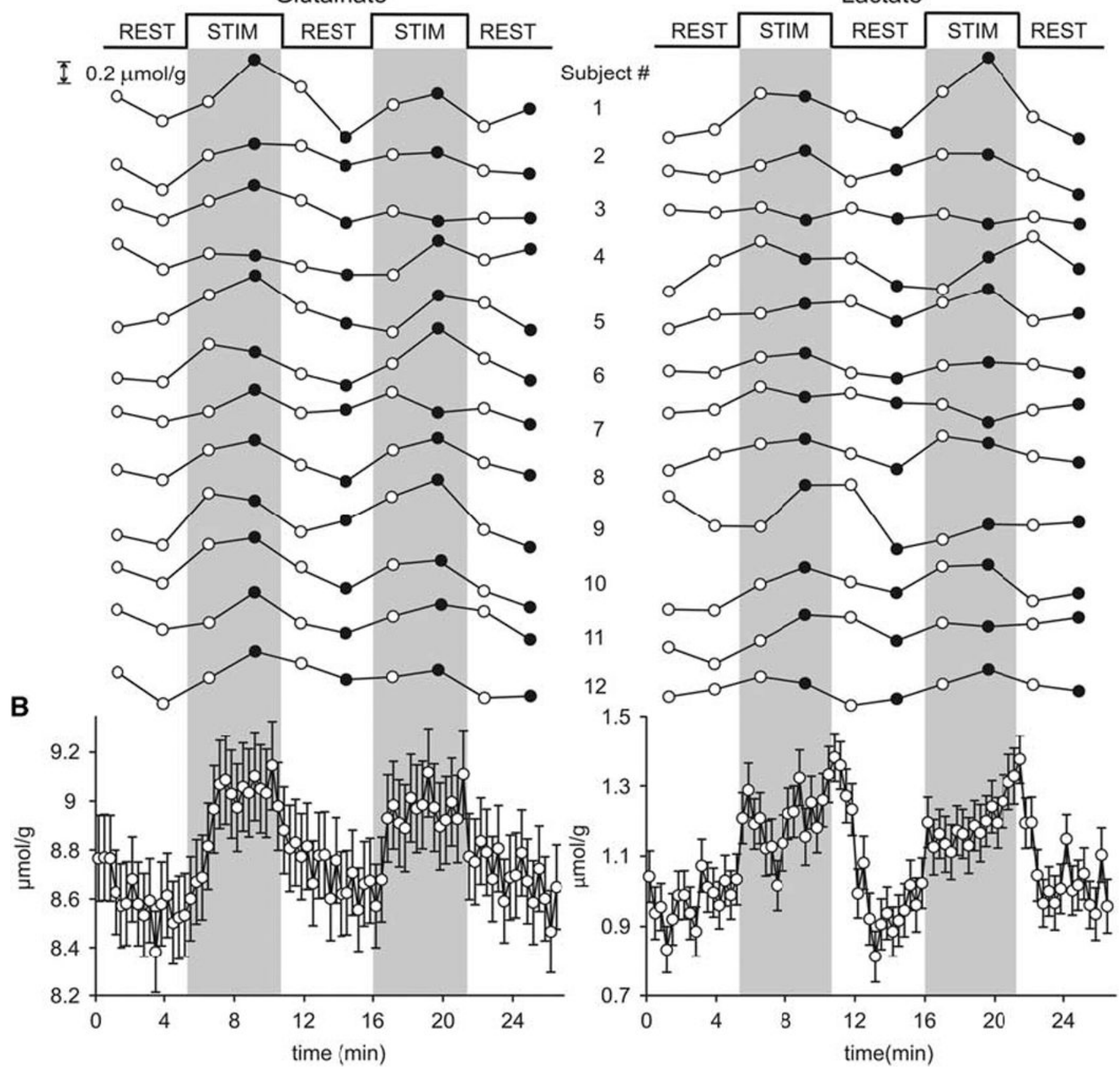

Figure 7.

(A) Time courses of glutamate and lactate concentrations during the visual stimulation paradigm (number of scans $=32,2.7$ minutes resolution) in individual subjects $(\mathrm{N}=12)$. The MRS data acquired in the second halves of the STIM and REST periods (black points) were used to calculate concentration differences between the STIM and REST conditions and for statistical analysis of fMRS data. (B) Time courses of glutamate and lactate concentrations with high temporal resolution. The fMRS data of four scans (20 seconds) were summed across all subjects $(\mathrm{N}=12)$. The resulting spectra $(4$ scans $\times 12$ subjects $=48$ scans per time 
point) were quantified by LCModel. The error bars indicate CRLBs. CRLBs, Cramèr-Rao lower bounds; fMRS, functional magnetic resonance spectroscopy; REST, resting condition; STIM, stimulation period ${ }^{59}$. 